\title{
In vivo phosphoproteomics reveals pathogenic signaling changes in diabetic islets
}

Francesca Sacco ${ }^{1,4^{*}}$, Anett Seelig ${ }^{2}$, Sean J. Humphrey ${ }^{3}$, Natalie Krahmer ${ }^{1}$, Francesco Volta ${ }^{2}$, Alessio Reggio ${ }^{4}$, Jantje Gerdes ${ }^{2}$, Matthias Mann ${ }^{1 *}$

1. Proteomics and Signal Transduction, Max-Planck Institute of Biochemistry, 82152 Martinsried, Germany

2. Helmholtz Diabetes Center (HMGU) and German Center for Diabetes Research (DZD), 85748 Garching, Munich, Germany

3. School of Life and Environmental Sciences, The University of Sydney, Sydney NSW 2006, Australia

4. Department of Biology, University of Rome Tor Vergata, 00100 Rome, Italy

*To whom correspondence may be addressed: fsacco@biochem.mpg.de, mmann@biochem.mpg.de,

\section{SUMMARY}

Progressive decline of pancreatic beta cells function is key to the pathogenesis of type 2 diabetes. Protein phosphorylation is the central mechanism controlling glucosestimulated insulin secretion in beta cells. However, if and how signaling networks are remodeled in diabetic islets in vivo remain unknowns. Here we applied highsensitivity mass spectrometry-based proteomics and quantified the levels of about 6,500 proteins and 13,000 phosphopeptides in islets of obese diabetic mice and matched controls. This highlighted drastic remodeling of key kinase hubs and signaling pathways. We integrated our phosphoproteomic dataset with a literature-derived signaling network, which revealed a crucial and conserved role of GSK3 kinase in the control of the beta cells-specific transcription factor PDX1 and insulin secretion, which we functionally verified. Our resource will enable the community to investigate potential mechanisms and drug targets in type 2 diabetes.

\section{INTRODUCTION}

The metabolic syndrome is caused by a complex interplay between genetic, epigenetic and lifestyle factors, including physical activity and diet (Zimmet et al., 2001). In particular, type 2 diabetes (T2D) mellitus is a major public health issue, accompanied by a chronic decline of glycemic control. The pathophysiology of type 2 diabetes starts with insulin resistance and proceeds to beta cell dysfunction (Prasad and Groop, 2015). Growing evidence implicates beta cells dysfunction as a key event during T2D development (Saisho, 2015). Genome-wide association (GWAS) studies have identified multiple risk variants for T2D with a primary role in beta cell function, highlighting their importance in the development of T2D (McCarthy and Hattersley, 2008). However, our knowledge of the molecular mechanisms underlying the development of beta cell dysfunction is still far from complete. A systems-wide understanding of beta cell dysregulation obtained by unbiased methods could contribute to the treatment and prevention of this disease. Large-scale 
"omics" technologies, particularly transcriptomics but also mass spectrometry (MS)-based proteomics, have been applied to characterize islets isolated from different T2D animal models and human cadavers (El Ouaamari et al., 2015; Hou et al., 2017; Lu et al., 2008; Segerstolpe et al., 2016). While these analyses provided sets of differentially expressed genes and proteins involved in beta cell failure, by their design they did not capture the crucial signaling alterations at the level of phosphorylation networks. As phosphorylation is a major regulator of insulin secretion and can also be modulated pharmacologically, its global investigation would be highly desirable.

Phosphorylation can be measured at a large scale by proteomics methods, but this involves specific enrichment for phosphorylated peptides and typically requires hundred-fold larger input material than proteomics measurements. Consequently, a major obstacle to the global characterization of changes in T2D islets by phosphoproteomics has been the extremely limited amount of material that can be extracted from pancreatic islets. Our group has recently described a MS-based phosphoproteomics workflow, termed 'EasyPhos', which enables streamlined and large-scale phosphoproteome analysis over multiple experimental conditions (Humphrey et al., 2015). Very recently, we have made further developments to this method, improving its sensitivity several-fold (Humphrey et al., 2018). We reasoned that this workflow might enable the in-depth characterization of changes in signaling networks of islets isolated from diabetic compared to control mice. For this purpose, we employed mice that carry an autosomal recessive mutation in the leptin receptor, the standard model in the field. These $\mathrm{Lepr}^{\mathrm{db} / \mathrm{db}}$ mice become obese from 3-4 weeks of age, and develop hyperglycamia as early as $4-8$ weeks (Chen et al., 1996). To obtain a comprehensive view of global signaling network rewiring, we combined the phosphoproteomic analysis of diabetic islets with an in-depth characterization of the proteome changes. Our analysis revealed a drastic remodeling of the proteome and phosphoproteome, including numerous proteins implicated in insulin secretion control, glucose uptake and metabolism. To functionally interpret our datasets we applied a recently developed bioinformatics workflow (Sacco et al., 2016b). This highlighted a novel GSK3-dependent signaling axis contributing to beta cell failure, whose molecular mechanism we investigated in mouse and rat cells as well as in human islet models.

\section{RESULTS}

\section{In-depth proteomic and phosphoproteomic characterization of $\mathbf{d b} / \mathbf{d b}$ islets}

To obtain a comprehensive perspective of the mechanisms underlying the insulin secretion failure occurring in diabetic islets, we measured the proteome and phosphoproteome of pancreatic islets isolated from 13 week old homozygous C57BLKS-Lepr ${ }^{\mathrm{db}}$ mice. As expected, these "db/db" mice were obese and hyperglycaemic (Fig. S1A-B). As negative controls, we used age-matched C57BLKSLepr $^{\mathrm{db}} /+$, because these heterozygous mice have the same genetic background (hereafter referred to as "Ctrl") (Fig. 1A).

We applied a label-free quantification approach followed by high-resolution LC-MS performed in a single-run format on the newest generation $Q$ Exactive mass spectrometers (Kelstrup et al., 2018; Kulak et al., 2014). Combined with the latest developments of our EasyPhos workflow, this allowed us to work on single mice for the proteome, whereas we harvested islets from three mice for the phosphoproteome 
measurements, yielding $200 \mu \mathrm{g}$ of islet proteins. This experimental strategy enabled us to reliably quantify 6,500 proteins (Fig. 1A, Supplementary Table S1) and more than
14,000 different phosphorylation events mapping to 3,821 proteins (Table S2; Fig. S2A-B). In total $88 \%$ of the phosphoproteome were localized with high

A
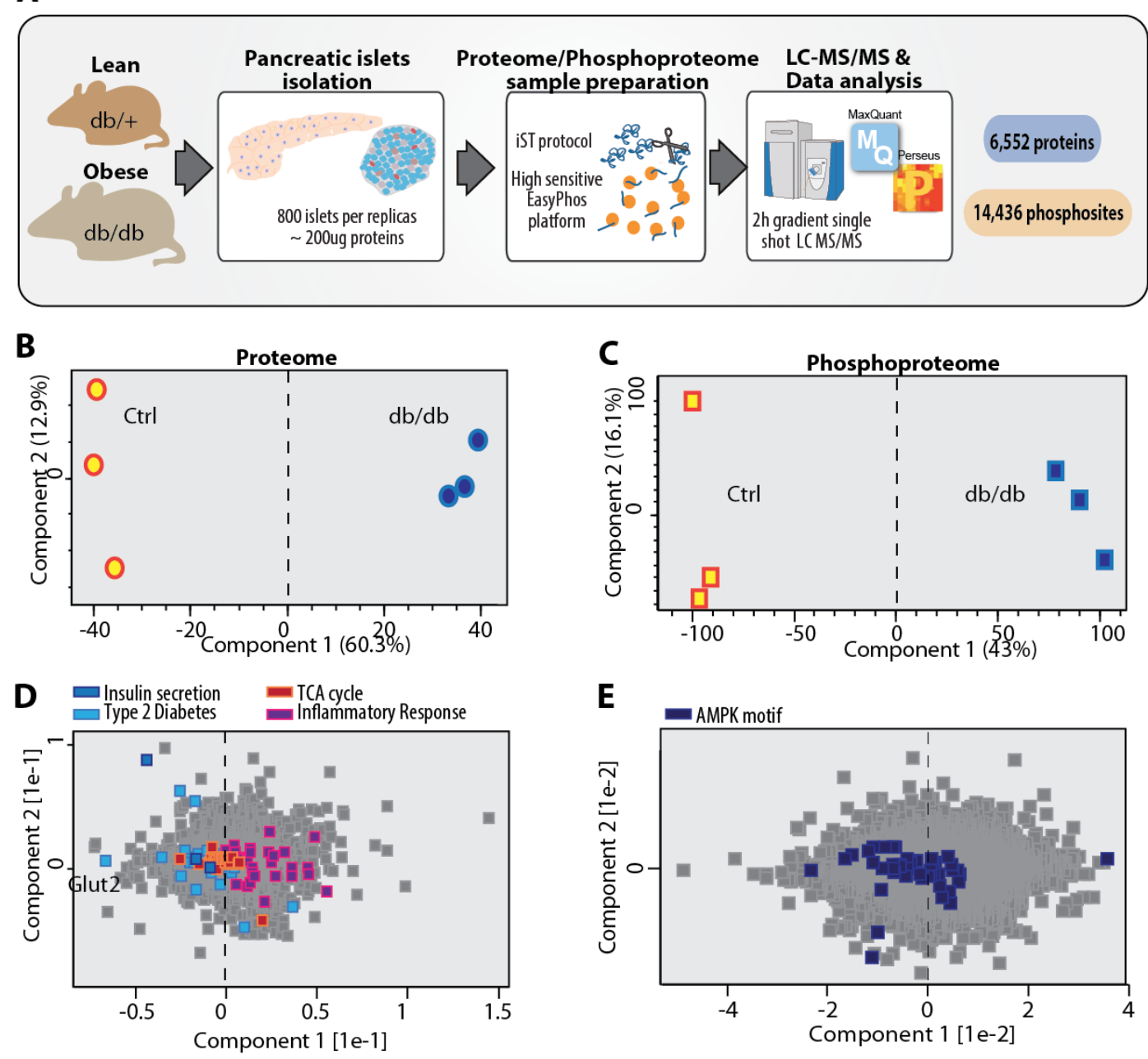

$\mathbf{F}$

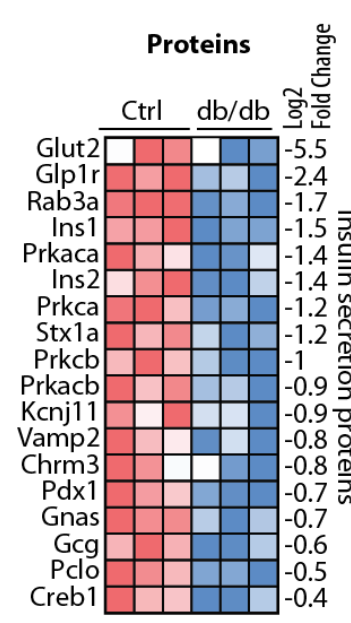

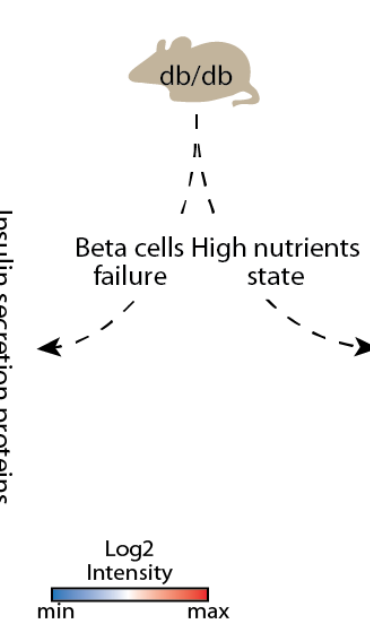

Figure 1. Our experimental strategy. (A) Experimental workflow to analyze the proteome and phosphoproteom of pancreatic islets derived from diabetic $(\mathrm{db} / \mathrm{db})$ and control $(\mathrm{db} /+)$ mice. Principal component analysis $($ PCA) of proteom (B) and phosphoproteom $(\mathbf{C})$ data. The loadings of the PCA in B and C reveal that the proteins (D) and phosphosites (E) responsible for driving the segregation in component 1 are significantly enriched in the GO BPs and Kegg pathways and kinase substrate motifs indicated $(\mathrm{FDR}<0.07)$. F) Heat map of protein levels of the labeled insulin secretion-associated proteins in control and $\mathrm{db} / \mathrm{db}$ islets upon drug and glucose treatments. (G) Heat map of phosphorylation levels of the indicated phosphosites in control and $\mathrm{db} / \mathrm{db}$ islets upon drug and glucose treatments. 
confidence to a single amino acid sequence location (Fig. S2C), with a median localization probability greater than 0.99 (Experimental Methods). These are excellent values, especially when considering the minimal amount of in vivo material, and the fact that no exogenous stimulation was employed.

Both proteome and phosphoproteome measurements where highly accurate and reproducible, with Pearson correlation coefficients of biological replicates ranging between 0.85 (lowest for phosphoproteome measurements) and 0.99 (highest for proteome measurements) (Fig. S2D-E). In agreement with other MS-based studies (Sharma et al., 2014), the majority of phosphorylation events in pancreatic islets occurred on serine residues $(88 \%)$, followed by threonine (11\%), whereas phosphotyrosines accounted for less than $1 \%$ of quantified phosphosites. Compared to the community database PhosphoSitePlus (Hornbeck et al., 2015), 87\% of our quantified phosphosites were identical - a reassuring proportion considering the small sample amount and the depth of the analysis (Figure S3A-B). Only 268 out of these 10,994 sites are annotated as 'regulatory', meaning that the functional role or upstream cognate kinases of the vast majority of phosphorylation events quantified here have not been investigated to date.

Principal component analysis (PCA) of both our proteomic and phosphoproteomic data clearly classified pancreatic islets according to their diabetic status (Fig. 1B-C). AMPK inhibition, mTOR hyperactivation and insulin secretion failure are features of type 2 diabetic islets (Blandino-Rosano et al., 2012; Kahn et al., 2006). The drivers of the discrimination (component 1 of the PCA loadings) at the proteome and phosphoproteome level are enriched in annotations with these processes, providing a positive control for our proteomics measurements (Fig. 1D-E). Likewise, $\mathrm{db} / \mathrm{db}$-upregulated proteins were also significantly enriched for inflammation processes, which is known to correlate with obesity and T2D progression (Donath et al., 2013). Additionally, key proteins involved in insulin secretion were significantly downregulated in $\mathrm{db} / \mathrm{db}$ islets with fold-changes between 0.5 and five-fold whereas the phosphorylation of almost all the mTOR/S6K substrates was increased by similar amounts (FDR < 0.05; Fig. 1F-G). We conclude that our proteomic screen recapitulated known biology of diabetic islets - in particular with regards to the effects of chronic high nutrients on mTOR hyperactivaton and insulin secretion failure - while generating a vast resource of previously unknown, regulated events.

We next compared our dataset to previous proteomic studies characterizing proteome remodeling in pancreatic islets of murine and rat T2D models. In insulin-resistant MKR mice, only 159 regulated proteins were reported (Lu et al., 2008), however, $45 \%$ of them were also regulated in our much deeper dataset (Fig. S4A). Likewise, in a study of $\mathrm{ob} / \mathrm{ob}$ and high-fat diet mice (El Ouaamari et al., 2015) 198 of the 272 significantly regulated proteins were likewise in our set. In addition to highly significant overlap of identifications, the Pearson correlation coefficients to our study were also relatively high (from 0.7 to 0.83) (Fig. S4B and Fig. S5A-D). In contrast, the 2,372 regulated proteins in non-obese GK rats (Hou et al., 2017) had less, although still statistically significant overlap with our data $(35 \%$; $p<$ $0.05)$. We attribute this to the fact that the previous models all involve obesity and insulin resistance, whereas beta-cell failure is apparently quite different in the GK model. Interestingly, a large fraction of the 107 genes significantly modulated in islets derived from all four different T2D models were involved 
Figure 2. Key signaling pathways are modulated in db/db islets. (A) Kinase substrates motifs significantly overrepresented in significantly modulated phosphosites in $\mathrm{db} / \mathrm{db}$ islets (Benjamin Hochberg FDR $<0.05)$. B) $\log 2$ fold change of the expression levels of the indicated kinases in $\mathrm{db} / \mathrm{db}$ islets with respect to control islets. C) The phosphoproteome and proteome changes observed in $\mathrm{db} / \mathrm{db}$ islets were mapped onto $\mathrm{a}$ literature curated signaling network. Node size is proportional to the protein expression change in $\mathrm{db} / \mathrm{db}$ islets. Blue and red nodes are inactivated or activated in $\mathrm{db} / \mathrm{db}$ islets, respectively. Phosphorylation and dephosphorylation reactions on specific residues are represented as edges between nodes. Phosphosites are colored according to their phosphorylation state in $\mathrm{db} / \mathrm{db}$ islets, as indicated in the legend.
A
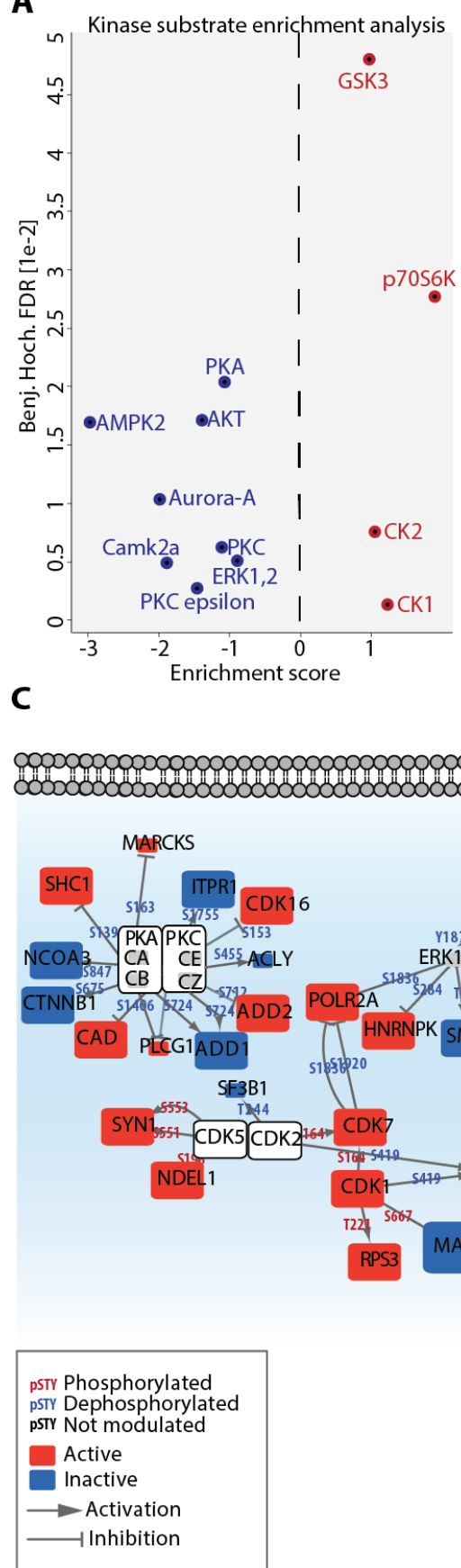

in the ER stress response (FDR $<0.05$; Fig. S4C and S5E). Glucolipotoxicity triggers the accumulation of misfolded proteins that are removed from the ER and subjected to endoplasmic reticulum-associated degradation (ERAD). Misfolded proteins are translocated to the cytosol, ubiquitylated and degraded in the proteasome (Karunakaran et al., 2012). Thus, these unbiased proteomic screens suggest that the upregulation of different components of the ubiquitin/proteasome system is a major common adaptation

\section{B}

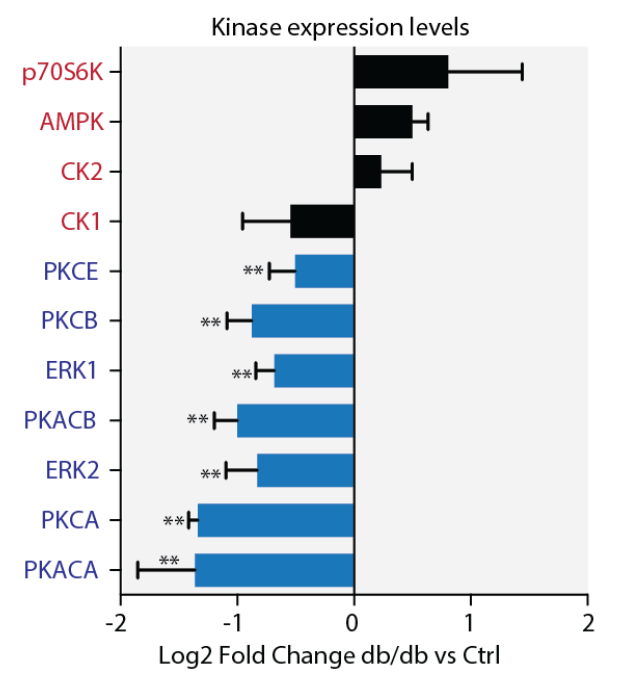


quantified at the protein level by our screen were significantly modulated (Fig. S4D). This suggests that protein levels might in some cases be linked to DNA variants of the respective gene products by affecting gene expression, protein stability, or degradation. This connection also implies that some of the proteins differentially expressed in the islets of this mouse model of T2D may also be affected in islets of T2D patients.

\section{Drastic remodeling of the proteome and phosphoproteome in $\mathbf{~} \mathrm{db} / \mathrm{db}$ islets}

We next investigated to what extent the proteome and phosphoproteome are altered in dysfunctional islets in our $\mathrm{db} / \mathrm{db}$ mice. Around $35 \%$ of the proteome were modulated in $\mathrm{db} / \mathrm{db}$ islets at an FDR $<0.07$ (Fig. S6A, S7A and Table S1) and more than $25 \%$ of phosphopeptides on over $40 \%$ of phosphoproteins (Fig. S6B, S7B-C and Table S2). Interestingly, the levels of $72 \%$ of these phosphopeptides are increased in $\mathrm{db} / \mathrm{db}$ with respect to control islets. We interpret this observation to reflect a more active global signaling due to the high nutrition state.

Combined analysis of the phosphoproteome and proteome data showed that phosphorylation sites were evenly detected, irrespective of protein abundance and that for $82 \%$ of the quantified phosphorylation sites, the corresponding protein was also quantified (Fig. S3C, D, S7D). When normalizing for the protein levels, more than $60 \%$ of sites were still significantly regulated, while the regulation of the remaining proteins was due to a combination of changing phosphorylation and protein levels. In $65 \%$ of those case, phosphorylation and protein levels both increased (46\%) or decreased (18\%) (Fig. S7E). Together, our data reveals that a full third of the proteome and a quarter of the phosphoproteome are changed in our diabetes model. This result also highlights the need for joint analysis of protein and phosphorylation changes for a complete view of the molecular changes underlying pancreatic islet dysfunction.

We next asked which biological processes were most altered in $\mathrm{db} / \mathrm{db}$ islets and at which molecular level. For the proteome, a strong down-regulation of proteins involved in insulin secretion (at least two-fold and up to 100-fold) correlated with an impairment of mitochondrial metabolic processes, such as TCA cycle and OXPHOS, and with an increased lipid metabolism (Fig. S7F). Downregulation of annotated insulin secretion proteins was also associated with increased ER and oxidative stress. Interestingly, $\mathrm{db} / \mathrm{db}$ islets showed a positive enrichment of proteins involved in cell-cell adhesion and cell migration. At the signaling level, the mTOR pathway and autophagy, a downstream process, were positively enriched in $\mathrm{db} / \mathrm{db}$ islets. These changes occurred only at the phosphoproteome level and will be discussed in more detail below.

Figure 3. PDX1 down-regulation leads to GLUT2 suppression and glycolysis inhibition. (A) Schematic representation of the molecular mechanisms leading to suppression of PDX1 and decreased expression of PDX1 targets (bar graph). (B) Schematic representation of glycolysis-TCA-OXPHOS-insulin exocytosis pathways. Down-regulated enzymes are shown as blue squares, while not modulate proteins are grey. 


\section{A}

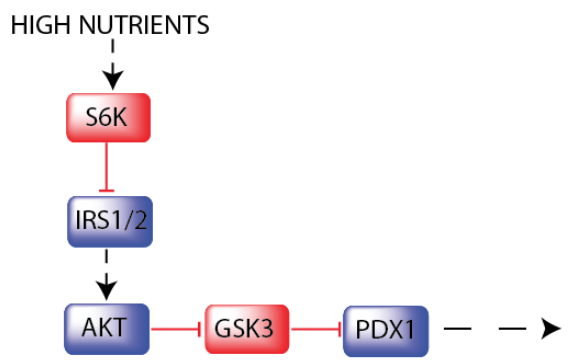

B
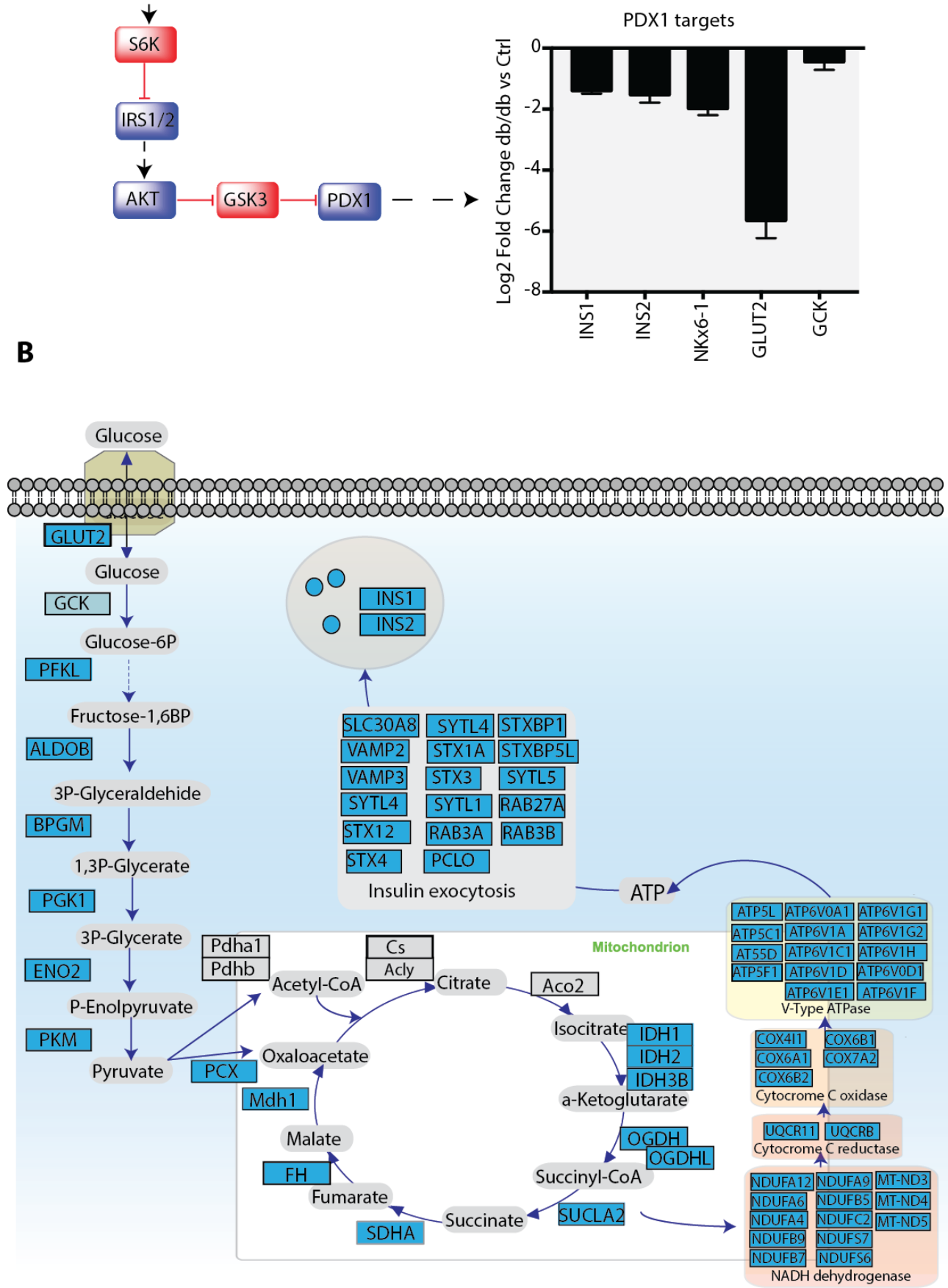

Key signaling pathways are remodeled in diabetic islets

To investigate the mechanisms responsible for the phosphoproteome level changes, we extracted the kinase substrate motifs (Keshava Prasad et al., 2009) significantly changed in $\mathrm{db} / \mathrm{db}$ islets (Fig. 2A) (Fisher exact test, FDR $<0.05$; see Experimental Procedures). Consistent with high nutrient-induced mTOR hyperactivation, the substrate motif of p70S6K was enriched, while the AMPK motif was down-regulated. Other key kinase motifs, such as those of ERK1/2 and AKT, were also down-regulated. Additionally, the substrate motifs of key kinases previously implicated in the regulation of insulin secretion, including PKA, PKC and CaMK (Sacco et al., 2016a; Shibasaki et al., 2007), were down-regulated, further explaining the inability of diabetic beta cells to properly secrete insulin after glucose stimulation. Enrichment or depletion of a specific substrate motif may be the consequence of the modulation of kinase activity or concentration and the proteome 
levels can help to unravel this question. We observed all of the catalytic subunits of PKA and $\mathrm{PKC}$ to be consistently down-regulated in $\mathrm{db} / \mathrm{db}$ islets, as well as the MAPKs ERK1 and ERK2 (Fig. 2B).

As our data clearly indicate that key kinases are modulated in $\mathrm{db} / \mathrm{db}$ islets, we next wanted to understand how signaling pathways are globally rewired in conditions of beta cells failure. We previously developed an approach that integrates large-scale quantitative phosphoproteomic and proteomic data with a literature-derived, proteome-wide signaling network (Sacco et al., 2016b). Exploration of the activation/inactivation pattern in the resulting graph confirmed and extended our previous observations, highlighting that all PKA and PKC substrates in our dataset were significantly dephosphorylated in the $\mathrm{db} / \mathrm{db}$ islets, and that the expression levels of the PKA and PKC catalytic subunits was also decreased (Fig. 2C). Several G protein-coupled receptors, including Gpr119, Glp1 and Gpr40, have attracted considerable interest as T2D drug targets, due to their potential positive effects on insulin secretion via indirect activation of PKA and PKC (Bailey et al., 2016). Our data indicate that PKA and PKC are downregulated, strongly suggesting that drug efficacy considerations should take the results of proteomics measurements into account.

Interestingly, we found that phosphorylation of the activating residue Thr8 of the SMAD2 transcription factor, which leads to its inactivation (Funaba et al., 2002), was significantly decreased. Consistently, Smad $2 \beta K O$ mice have striking islet hyperplasia together with defective glucoseresponsive insulin secretion (Nomura et al., 2014).

The high-nutrient-induced mTOR hyperactivation that we observed in diabetic islets leads to inhibition of AKT through the well-characterized p70S6K-IRS1/2 negative feedback loop (Hsu et al., 2011). Decreased AKT activity in turn decreases phosphorylation of its direct substrate, the inhibitory Ser9 of GSK3, which we indeed observed in our dataset. Likewise, phosphorylation of the activating Tyr216 of GSK3 was significantly increased, further confirming increased GSK3 activity in $\mathrm{db} / \mathrm{db}$ islets. Ser269 of the key beta cell specific transcription factor PDX1 is a direct substrate of GSK3, and phosphorylation of this residue causes its proteasomal degradation (Humphrey et al., 2010). In $\mathrm{db} / \mathrm{db}$ islets the phosphorylation of the Ser269 of PDX1 was increased, while the protein level of PDX1 impaired (Fig. S8). Together, our joined literature and proteomic network, suggests a signaling axis starting from mTOR hyperactivation, GSK3 activation and PDX1 degradation, whose functional role in diabetic islets we investigate below.

\section{PDX1 suppression down-regulates GLUT2 and inhibits glycolysis in $\mathbf{d b} / \mathbf{d b}$ islets}

Given our finding that GSK3 is hyperactivated in diabetic islets, leading to PDX1 degradation and the established importance of PDX1 in beta cell physiology, we asked whether the expression of downstream targets of PDX1 was also impaired in beta cells. Such targets include the glycolytic enzyme glucokinase, the insulin genes, the major murine islet glucose transporter GLUT2, and the two transcription factors NKX6.1 and HNF4A (Gao et al., 2014). Remarkably, all of these PDX1 targets were significantly down-regulated in $\mathrm{db} / \mathrm{db}$ islets (except for HNF4A, which we did not identify), with GLUT2 protein levels drastically decreasing by 64 -fold in $\mathrm{db} / \mathrm{db}$ islets compared to control islets (Fig. 3A). In rodents, GLUT2 is the only glucose transporter detected in normal pancreatic islets and therefore it has a crucial role in the insulin secretion pathway. In the absence of 
A

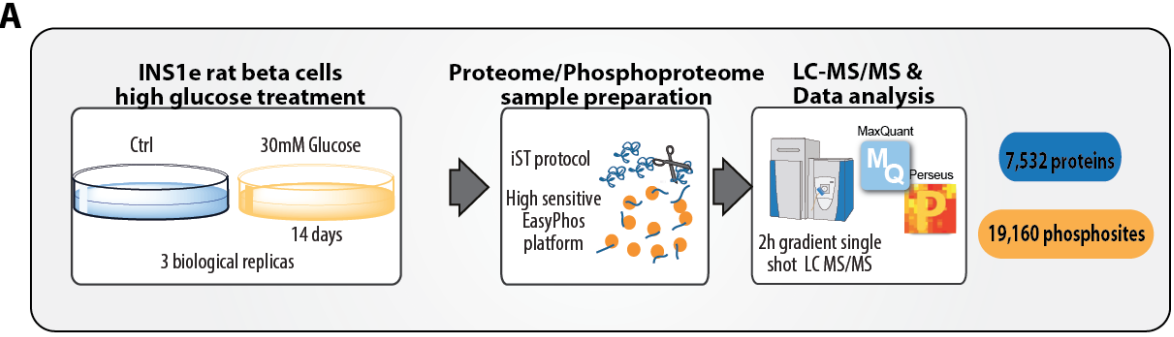

B
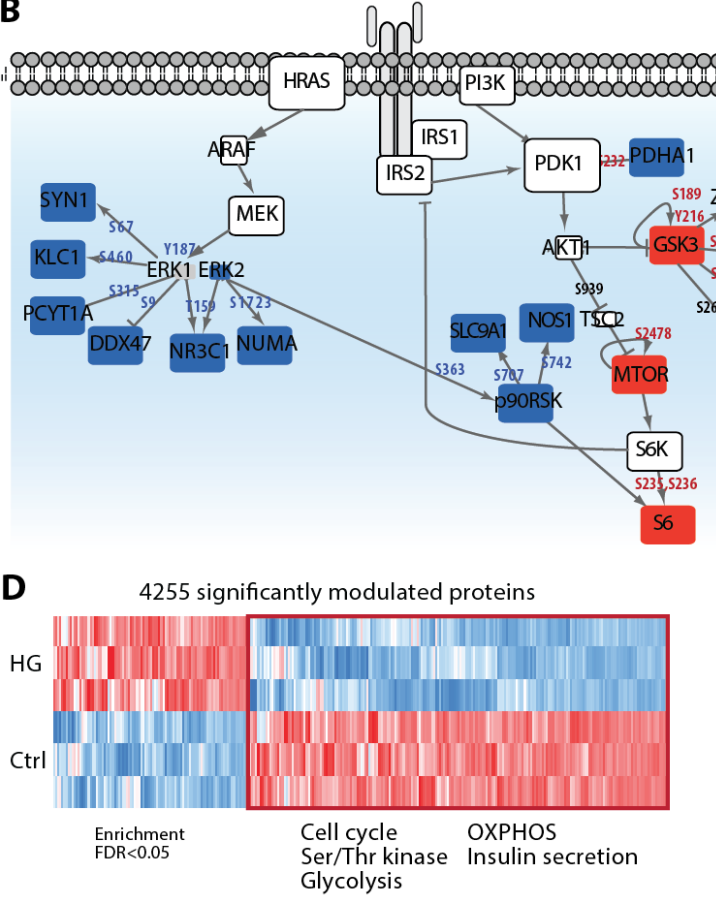

$\mathbf{E}$

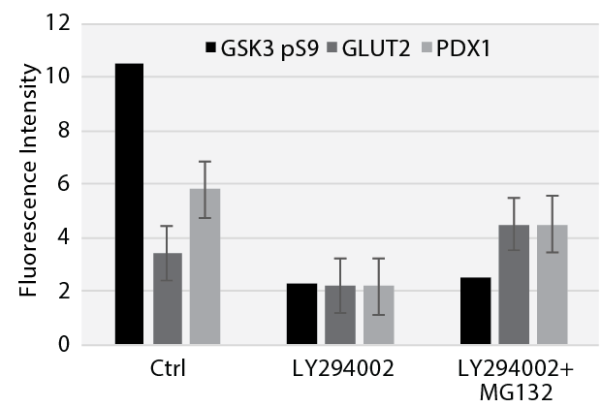

E

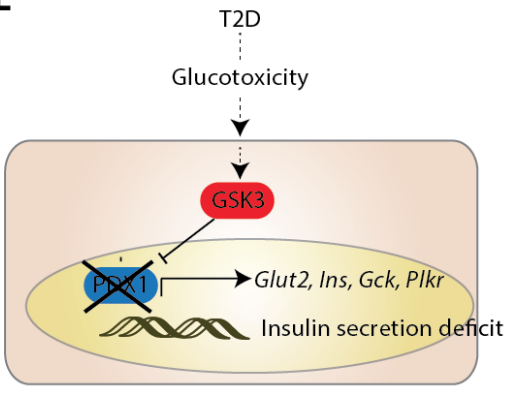

Figure 4. Glucotoxicity triggers GSK3-dependent PDX1 degradation. A) Schematic representation of the experimental workflow applied to analyze the proteome and phosphoproteome of INS1e cells after chronic high glucose stimulation. B) Proteome and phosphoproteome datasets were overlaid onto a literature-derived signaling network. C) Log2 protein expression fold change of PDX1 and its transcriptional targets. D) Heatmap of the significantly modulated proteins in INS1e cells after chronic high glucose stimulation. Significantly enriched GO terms and pathways are indicated $(\mathrm{FDR}<0.07)$. $\mathbf{E})$ Immunofluorescent signal of anti-GSK3 (P), anti-GLUT2 and anti-PDX1. F) A proposed model contributing to glucotoxicitydependent beta cell failure

GLUT2, there is no compensatory expression of either GLUT1 and GLUT3 in islets (Guillam et al., 2000), and accordingly, our in-depth proteomic analysis detected neither GLUT1 nor GLUT3. Decreased expression of GLUT2 strongly impairs glucose ability to effect insulin biosynthesis and secretion and this correlates with a strong impairment of whole body glucose utilization (Guillam et al., 2000). Consistently, our combined proteomic and literature mining approach revealed that in $\mathrm{db} / \mathrm{db}$ islets most of the key enzymes involved in glycolysis and the TCA cycle were significantly down-regulated, globally impairing glucose metabolism (Fig. 3B). In particular, we observed that the expression levels of almost all the subunits of the respiratory enzymes were also significantly decreased - by two-fold on average. Upon increased plasma glucose concentrations, this will impair a corresponding rise of ATP and the lower $[\mathrm{ATP}] /[\mathrm{ADP}]$ concentration should negatively affect downstream components of the insulin secretion machinery. Indeed, the proteomics data indicated a significant downregulation of the protein level of the $\mathrm{K}_{\mathrm{ATP}}$ channel subunits 
(1.5-fold, Table S1). Thus our data provide a molecular explanation for the insulin secretion failure observed in islets from 10-13 weeks old $\mathrm{db} / \mathrm{db}$ mice (Do et al., 2014; Guan et al., 2016; Kim et al., 2015; Kondo et al., 2012).

\section{Glucotoxicity triggers the GSK3-dependent down-regulation of PDX1}

During T2D, different factors synergistically contribute to beta cell loss and dysfunction. Chronic or recurrent exposure of beta cells to elevated levels of glucose and lipids (glucolipotoxicity) or to proinflammatory cytokines interfere with beta cell function, contributing to their destruction (Prentki and Nolan, 2006). Given the key role of GSK3 in glucose homeostasis (Humphrey et al., 2010) and having demonstrated that GSK3 inhibits PDX1 and that PDX1 suppression leads to an impairment of insulin secretion machinery in $\mathrm{db} / \mathrm{db}$ islets, we next aimed to investigate whether glucotoxicity in isolation triggers GSK3 activation in beta cells leading to consequent degradation of PDX1 transcription factor and its targets.

To address this question, we chronically treated isolated INS1e rat beta cells with high glucose and applied our MS-based proteomic and phosphoproteomic workflows to study global changes in these cells (Fig. 4A). These experiments enabled the reproducible quantification of around 7,500 proteins and 19,000 phosphosites and their response to chronically elevated glucose levels (Fig. S9BC, Table S3). In this experimental system, glucotoxicity drastically affected both the proteome and phosphoproteome, modulating the expression of 4,255 proteins and the phosphorylation level of 5,334 phosphopeptides (Fig?). To investigate and interpret the activation status of the PI3KAKT-GSK3 and other key signaling pathways, we again integrated our proteomic and phosphoproteomic dataset with the literature-derived signaling network. This revealed that chronic hyperglycaemia in rat beta cells also leads to GSK3 hyperactivation, as specifically demonstrated by six-fold increased phosphorylation of the activating Tyr216 of GSK3. We also observed the subsequent PDX1 phosphorylation by GSK3, causing its proteasomal degradation, as evidenced by its decreased protein levels in our proteomics data (Fig. 4B). Consistently, the expression levels of all the identified PDX1 targets, including GLUT2, INS, GCK and PLKR, were also decreased (Fig. 4C). Confirming out results in the $\mathrm{db} / \mathrm{db}$ islets, PDX1 suppression was correlated with suppression of proteins involved in glucose metabolism, TCA cycle, oxidative phosphorylation and insulin secretion (Fig. 4D).

To further assess the functional role of GSK3 in the regulation of PDX1 stability and its transcriptional target GLUT2, we treated human islets with a PI3K inhibitor to trigger GSK3 activation alone or in combination with the proteasome inhibitor MG132. Confirming our hypothesis, GSK3 activation was sufficient to cause PDX1 degradation and GLUT2 suppression (Fig. 4E, Fig. S10A). Importantly, GSK3 activation also impaired glucose stimulated insulin secretion (Fig. S7B). These effects were completely abrogated by co-treatment with the proteasome inhibitor.

The global proteomics and phosphoproteomic data, together with our functional follow up, thus support a model whereby a diabetic environment, including chronic hyperglycemia and associated glucotoxicity leads to beta cell failure in part through a GSK3-PDX1 dependent axis (Fig. 4E).

\section{Conclusion}

Complex diseases are rarely caused by an abnormality in a single gene, but rather by perturbations of global cellular networks. 
Investigating signaling networks rewiring in diabetic islets has been a major goal of the scientific community, however, the extremely limited amount of material that can be extracted from these structures composed of just a few thousand cells have prevented this so far. Combining a newly developed, highlysensitive phosphoproteomic workflow with MS-based proteomics we here obtained a first and highly-comprehensive landscape of dynamic changes in protein expression and phosphorylation in obese diabetic mice.

Integrating our large-scale dataset with literature-derived signaling pathways enabled insights into how miss-regulated proteins and signaling events contribute to islets dysfunction. Here we focused on the mechanisms hyperactivation of the key kinase GSK3 in $\mathrm{db} / \mathrm{db}$ islets, further characterizing its functional role in insulin secretion regulation through a PDX1-dependent mechanism. Tus our proteomic data provide a molecular explanation of the already discovered beneficial effects of GSK3 inhibitor drugs in T2D treatment (MacAulay and Woodgett, 2008; Stein et al., 2011). We hope that in-depth exploration of the resource provided here will aid in the discovery of other potential islet-related diagnostic and therapeutic targets for human T2D.

\section{Experimental procedure}

Islet isolation. 13 week old C57BLKSLeprdb homozygous and age-matched C57BLKS-Leprdb/+ heterozygous mice (Charles River Laboratories) were euthanized. The upper abdomen was incised to expose liver and intestines. The pancreas was perfused through the common bile duct with cold collagenase P (from Roche) in saline solution. The pancreas was dissected and placed into a warm collagenase saline solution for $15 \mathrm{~min}$. After enzymatic digestion of the pancreatic tissue, islet were picked and left to recover at $37^{\circ} \mathrm{C}$ for 2 hour.

Insulin assay. Cells were grown overnight with DMEM low glucose medium, then washed with Krebs-Ringer-Buffer and incubated with starvation buffer for $90 \mathrm{~min}$. Cells were then incubated with high glucose medium (Krebs-Ringer-Buffer supplemented with glucose $16.7 \mathrm{mM}$ and BSA $0.05 \%$ ) or low glucose medium (Krebs-Ringer-Buffer supplemented with glucose $2.5 \mathrm{mM}$ and BSA $0.05 \%$ ). Aliquots of the supernatant were assayed for the amount of insulin (insulin assay from Cisbio Bioassays), according to the manufacturer's protocol.

\section{Proteome and phosphoproteome sample} preparation. Cells were lysed in SDC lysis buffer containing 4\% (w/v) SDC, $100 \mathrm{mM}$ Tris $-\mathrm{HCl}(\mathrm{pH} 8.5)$. Proteome preparation was done using the in StageTip (iST) method (Kulak et al., 2014). Phosphoproteome preparation was performed as previously described (Humphrey 2015; Humphrey et al., 2018). Per condition, a total of only $200 \mathrm{ug}$ protein input material was lysed, alkylated and reduced in one single step. Then proteins were digested and phosphopeptides enriched by $\mathrm{TiO} 2$ beads. After elution, samples were separated by HPLC in a single run (without pre-fractionations) and analyzed by mass spectrometry.

Mass spectrometric analyses. The peptides or phosphopeptides were desalted on StageTips and separated on a reverse phase column (packed in-house with 1.8-mm C18Reprosil-AQ Pur reversed-phase beads) (Dr Maisch $\mathrm{GmbH}$ ) over $240 \mathrm{~min}$ or $270 \mathrm{~min}$ (single-run proteome and phosphoproteome analysis). Eluting peptides were electrosprayed and analyzed by tandem mass spectrometry on a Q Exactive HF (Thermo Fischer Scientific) using HCD based 
fragmentation, which was set to alternate between a full scan followed by up to five fragmentation scans. Proteome and phosphoproteome data were processed and statistically analyzed as described in Supplementary Methods.

Cell culture. INS-1E cells (RRID: CVCL_0351) were grown in a humidified atmosphere $\left(5 \% \mathrm{CO} 2,95 \%\right.$ air at $\left.37{ }^{\circ} \mathrm{C}\right)$ in

\section{Author contributions}

F.S. conceived and M.M. supervised the project. F.S. performed the proteome, phosphoproteome, immunofluorescence and insulin secretion analyses. A.S., F.V. dissected mice, isolated islets. N.K. measured the last batch of proteome samples. S.H.H optimized the workflow for phosphoproteome islets analysis. A.R. helped in the GSK3 activation experiments. F.S. analyzed the data. F.S., N.K, S.H.H, J.G., M.M. wrote the manuscript.

\section{Acknowledgements}

We thank Igor Paron, Korbinian Mayr for technical assistance. These studies were supported by the Max Plank Society for the Advancement of Science.

\section{REFERENCES}

Bailey, C.J., Tahrani, A.A., and Barnett, A.H. (2016). Future glucose-lowering drugs for type 2 diabetes. Lancet Diabetes Endocrinol 4, 350-359.

Blandino-Rosano, M., Chen, A.Y., Scheys, J.O., Alejandro, E.U., Gould, A.P., Taranukha, T., Elghazi, L., Cras-Meneur, C., and Bernal-Mizrachi, E. (2012). mTORC1 signaling and regulation of pancreatic betacell mass. Cell Cycle 11, 1892-1902.

Chen, H., Charlat, O., Tartaglia, L.A., Woolf, E.A., Weng, X., Ellis, S.J., Lakey, N.D., Culpepper, J., Moore, K.J., Breitbart, R.E., et monolayer in modified RPMI 1,640 medium supplemented with $10 \%$ fetal calf serum, 10 $\mathrm{mM}$ Hepes, $100 \mathrm{U} \mathrm{ml}^{-1}$, penicillin, $100 \mathrm{mg}$ $\mathrm{ml}^{-1}$, streptomycin, $1 \mathrm{mM}$ sodium pyruvate, $50 \mathrm{mM}$ b-mercaptoethanol (all from Gibco) and $0.5 \%$ BSA (from Sigma). Human islets were purchased from PELO Biotech and grown according to manufacturer instructions (CellProgen cat n. 35002-04).

al. (1996). Evidence that the diabetes gene encodes the leptin receptor: identification of a mutation in the leptin receptor gene in $\mathrm{db} / \mathrm{db}$ mice. Cell 84, 491-495.

Do, O.H., Low, J.T., Gaisano, H.Y., and Thorn, P. (2014). The secretory deficit in islets from $\mathrm{db} / \mathrm{db}$ mice is mainly due to a loss of responding beta cells. Diabetologia 57, 1400-1409.

Donath, M.Y., Dalmas, E., Sauter, N.S., and Boni-Schnetzler, M. (2013). Inflammation in obesity and diabetes: islet dysfunction and therapeutic opportunity. Cell Metab 17, 860872.

El Ouaamari, A., Zhou, J.Y., Liew, C.W., Shirakawa, J., Dirice, E., Gedeon, N., Kahraman, S., De Jesus, D.F., Bhatt, S., Kim, J.S., et al. (2015). Compensatory Islet Response to Insulin Resistance Revealed by Quantitative Proteomics. J Proteome Res 14, 3111-3122.

Funaba, M., Zimmerman, C.M., and Mathews, L.S. (2002). Modulation of Smad2mediated signaling by extracellular signalregulated kinase. J Biol Chem 277, 4136141368.

Gao, T., McKenna, B., Li, C., Reichert, M., Nguyen, J., Singh, T., Yang, C., Pannikar, A., Doliba, N., Zhang, T., et al. (2014). Pdx1 maintains beta cell identity and function by repressing an alpha cell program. Cell Metab 19, 259-271.

Guan, S.S., Sheu, M.L., Yang, R.S., Chan, D.C., Wu, C.T., Yang, T.H., Chiang, C.K., and Liu, S.H. (2016). The pathological role of 
advanced glycation end productsdownregulated heat shock protein 60 in islet beta-cell hypertrophy and dysfunction. Oncotarget 7, 23072-23087.

Guillam, M.T., Dupraz, P., and Thorens, B. (2000). Glucose uptake, utilization, and signaling in GLUT2-null islets. Diabetes 49, 1485-1491.

Hornbeck, P.V., Zhang, B., Murray, B., Kornhauser, J.M., Latham, V., and Skrzypek, E. (2015). PhosphoSitePlus, 2014: mutations, PTMs and recalibrations. Nucleic Acids Res 43, D512-520.

Hou, J., Li, Z., Zhong, W., Hao, Q., Lei, L., Wang, L., Zhao, D., Xu, P., Zhou, Y., Wang, Y., et al. (2017). Temporal Transcriptomic and Proteomic Landscapes of Deteriorating Pancreatic Islets in Type 2 Diabetic Rats. Diabetes 66, 2188-2200.

Hsu, P.P., Kang, S.A., Rameseder, J., Zhang, Y., Ottina, K.A., Lim, D., Peterson, T.R., Choi, Y., Gray, N.S., Yaffe, M.B., et al. (2011). The mTOR-regulated phosphoproteome reveals a mechanism of mTORC1-mediated inhibition of growth factor signaling. Science 332, 1317-1322.

Humphrey, R.K., Yu, S.M., Flores, L.E., and Jhala, U.S. (2010). Glucose regulates steadystate levels of PDX1 via the reciprocal actions of GSK3 and AKT kinases. J Biol Chem 285, 3406-3416.

Humphrey, S.J., Azimifar, S.B., and Mann, M. (2015). High-throughput phosphoproteomics reveals in vivo insulin signaling dynamics. Nat Biotechnol 33, 990995.

Kahn, S.E., Hull, R.L., and Utzschneider, K.M. (2006). Mechanisms linking obesity to insulin resistance and type 2 diabetes. Nature 444, 840-846.

Karunakaran, U., Kim, H.J., Kim, J.Y., and Lee, I.K. (2012). Guards and culprits in the endoplasmic reticulum: glucolipotoxicity and beta-cell failure in type II diabetes. Exp Diabetes Res 2012, 639762.

Kelstrup, C.D., Bekker-Jensen, D.B., Arrey, T.N., Hogrebe, A., Harder, A., and Olsen, J.V. (2018). Performance Evaluation of the Q Exactive HF-X for Shotgun Proteomics. J Proteome Res 17, 727-738.

Keshava Prasad, T.S., Goel, R., Kandasamy, K., Keerthikumar, S., Kumar, S., Mathivanan, S., Telikicherla, D., Raju, R., Shafreen, B., Venugopal, A., et al. (2009). Human Protein Reference Database--2009 update. Nucleic Acids Res 37, D767-772.

Kim, K.S., Jung Yang, H., Lee, I.S., Kim, K.H., Park, J., Jeong, H.S., Kim, Y., Ahn, K.S., Na, Y.C., and Jang, H.J. (2015). The aglycone of ginsenoside $\mathrm{Rg} 3$ enables glucagon-like peptide-1 secretion in enteroendocrine cells and alleviates hyperglycemia in type 2 diabetic mice. Sci Rep 5, 18325.

Kondo, T., Sasaki, K., Matsuyama, R., Morino-Koga, S., Adachi, H., Suico, M.A., Kawashima, J., Motoshima, H., Furukawa, N., Kai, H., et al. (2012). Hyperthermia with mild electrical stimulation protects pancreatic beta-cells from cell stresses and apoptosis. Diabetes 61, 838-847.

Kulak, N.A., Pichler, G., Paron, I., Nagaraj, N., and Mann, M. (2014). Minimal, encapsulated proteomic-sample processing applied to copy-number estimation in eukaryotic cells. Nat Methods 11, 319-324.

Lu, H., Yang, Y., Allister, E.M., Wijesekara, N., and Wheeler, M.B. (2008). The identification of potential factors associated with the development of type 2 diabetes: a quantitative proteomics approach. Mol Cell Proteomics 7, 1434-1451.

MacAulay, K., and Woodgett, J.R. (2008). Targeting glycogen synthase kinase-3 (GSK3 ) in the treatment of Type 2 diabetes. Expert Opin Ther Targets 12, 1265-1274. 
McCarthy, M.I., and Hattersley, A.T. (2008).

Learning from molecular genetics: novel insights arising from the definition of genes for monogenic and type 2 diabetes. Diabetes $57,2889-2898$.

Nomura, M., Zhu, H.L., Wang, L., Morinaga, H., Takayanagi, R., and Teramoto, N. (2014). SMAD2 disruption in mouse pancreatic beta cells leads to islet hyperplasia and impaired insulin secretion due to the attenuation of ATP-sensitive $\mathrm{K}+$ channel activity. Diabetologia 57, 157-166.

Prasad, R.B., and Groop, L. (2015). Genetics of type 2 diabetes-pitfalls and possibilities. Genes (Basel) 6, 87-123.

Prentki, M., and Nolan, C.J. (2006). Islet beta cell failure in type 2 diabetes. J Clin Invest 116, 1802-1812.

Sacco, F., Humphrey, S.J., Cox, J., Mischnik, M., Schulte, A., Klabunde, T., Schafer, M., and Mann, M. (2016a). Glucose-regulated and drug-perturbed phosphoproteome reveals molecular mechanisms controlling insulin secretion. Nat Commun 7, 13250.

Sacco, F., Silvestri, A., Posca, D., Pirro, S., Gherardini, P.F., Castagnoli, L., Mann, M., and Cesareni, G. (2016b). Deep Proteomics of Breast Cancer Cells Reveals that Metformin Rewires Signaling Networks Away from a Pro-growth State. Cell Syst 2, 159-171.

Saisho, Y. (2015). beta-cell dysfunction: Its critical role in prevention and management of type 2 diabetes. World J Diabetes 6, 109-124.

Scott, R.A., Scott, L.J., Magi, R., Marullo, L., Gaulton, K.J., Kaakinen, M., Pervjakova, N., Pers, T.H., Johnson, A.D., Eicher, J.D., et al. (2017). An Expanded Genome-Wide Association Study of Type 2 Diabetes in Europeans. Diabetes 66, 2888-2902.

Segerstolpe, A., Palasantza, A., Eliasson, P., Andersson, E.M., Andreasson, A.C., Sun, X., Picelli, S., Sabirsh, A., Clausen, M., Bjursell, M.K., et al. (2016). Single-Cell Transcriptome Profiling of Human Pancreatic
Islets in Health and Type 2 Diabetes. Cell Metab 24, 593-607.

Sharma, K., D'Souza, R.C., Tyanova, S., Schaab, C., Wisniewski, J.R., Cox, J., and Mann, M. (2014). Ultradeep human phosphoproteome reveals a distinct regulatory nature of Tyr and Ser/Thr-based signaling. Cell Rep 8, 1583-1594.

Shibasaki, T., Takahashi, H., Miki, T., Sunaga, Y., Matsumura, K., Yamanaka, M., Zhang, C., Tamamoto, A., Satoh, T., Miyazaki, J., et al. (2007). Essential role of Epac2/Rap1 signaling in regulation of insulin granule dynamics by cAMP. Proc Natl Acad Sci U S A 104, 19333-19338.

Stein, J., Milewski, W.M., Hara, M., Steiner, D.F., and Dey, A. (2011). GSK-3 inactivation or depletion promotes beta-cell replication via down regulation of the CDK inhibitor, p27 (Kip1). Islets 3, 21-34.

Zimmet, P., Alberti, K.G., and Shaw, J. (2001). Global and societal implications of the diabetes epidemic. Nature 414, 782-787. 


\section{Supplementary material for Sacco et al.}

\section{Supplementary Figures}

Figure S1. db/db mice are obese with respect to control mice. Body weight $(A)$ and plasma glucose (B) of $\mathrm{db} / \mathrm{db}$ and control 13 weeks old mice.

A

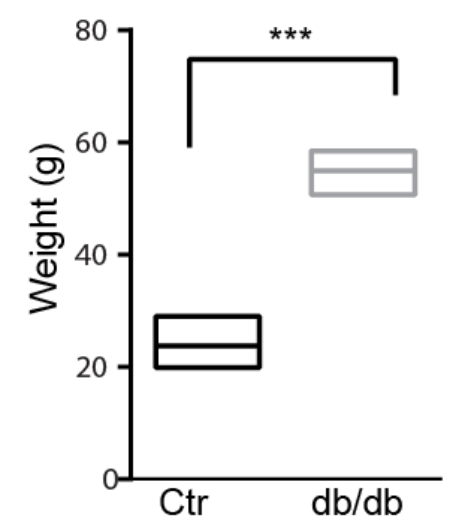

B

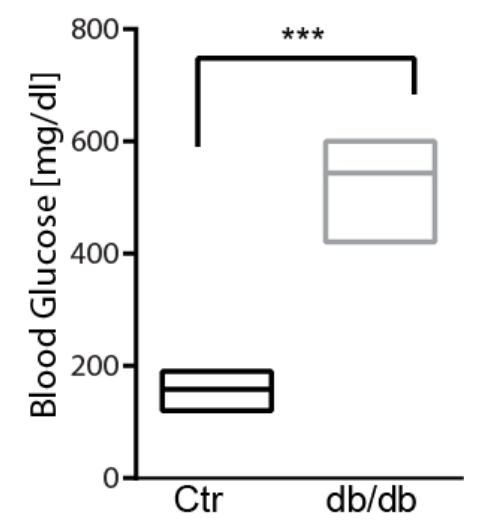


Figure S2. High coverage and reproducibility of proteome and phosphoproteome data. Number of quantified proteins (A) and phosphosites (B) in biological replicates of different experimental conditions. C) Venn diagram showing the proportion of class 1 sites in the whole quantified phosphosites. Heatmap showing the Pearson correlation coefficients between the different biological replicates in the proteome (D) and phosphoproteome (E) datasets.

A

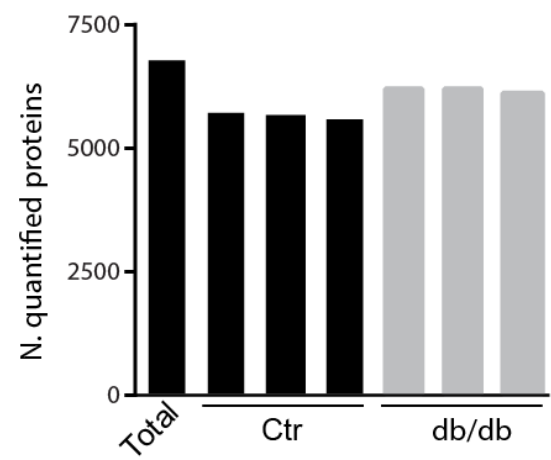

B

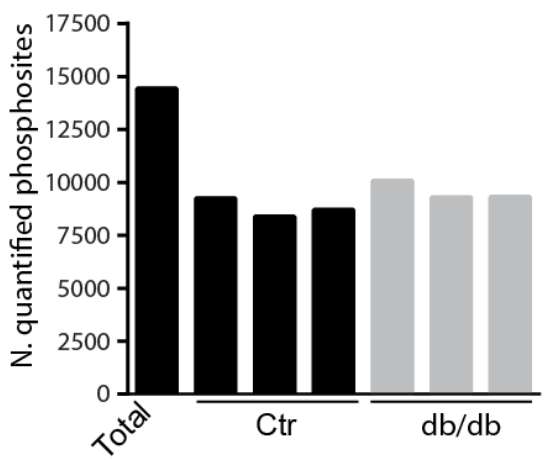

\section{C}

14,436 phosphosites

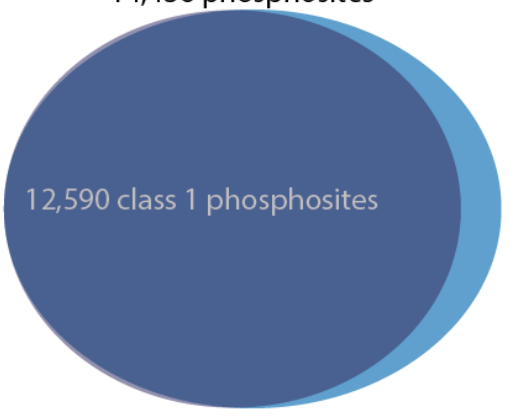

D

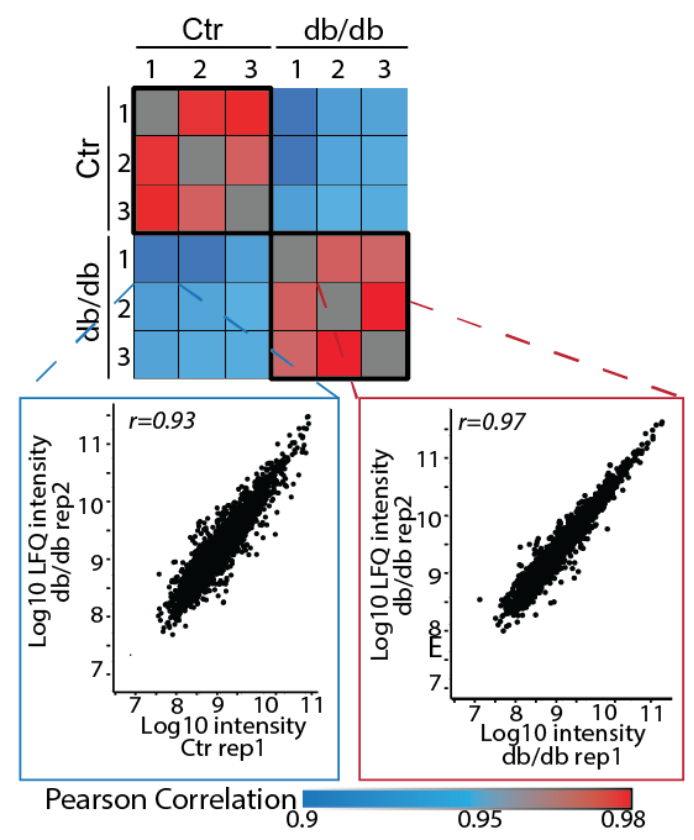

$\mathbf{E}$

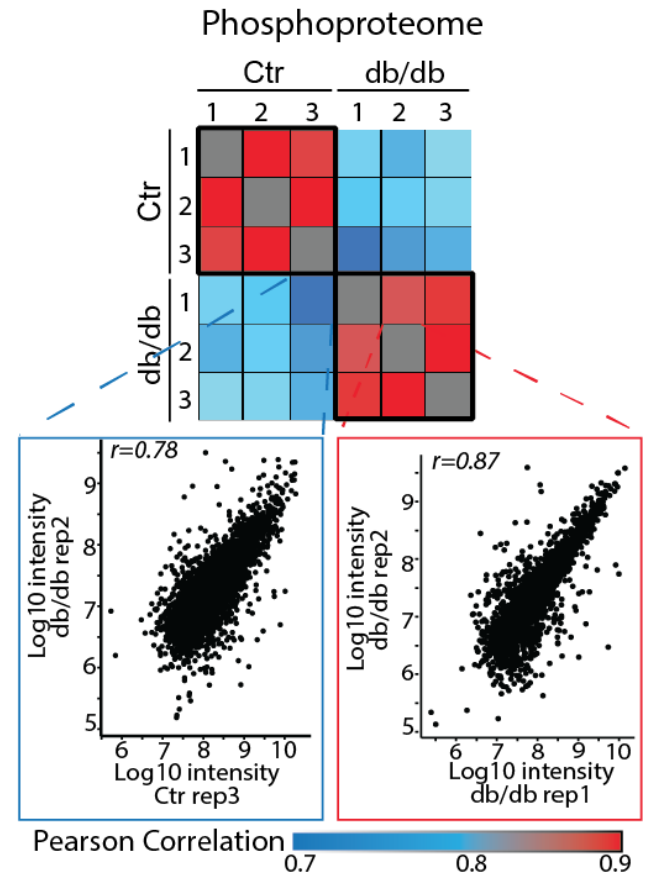


Figure S3. Data summary of the proteomic and phosphoproteomic data. A) Distribution of serine, threonine and tyrosine phosphorylation sites. B) Phosphosites quantified in our study that are already present in PhosphoSitePlus and annotated as "regulatory sites". Phosphorylated proteins are distributed in the entire range of measured protein abundances. The distribution of ranked $\log 2$ LFQ intensity values in control $(\mathbf{C})$ and $\mathrm{db} / \mathrm{db}$ islets $(\mathbf{D})$ are colour coded in grey, while phosphorylation intensity is in blue. E) Plot showing the distribution of the amplitudes (fold change of the $\log 2$ intensities) calculated for the phosphoproteome (blue) as well as for proteome.

A

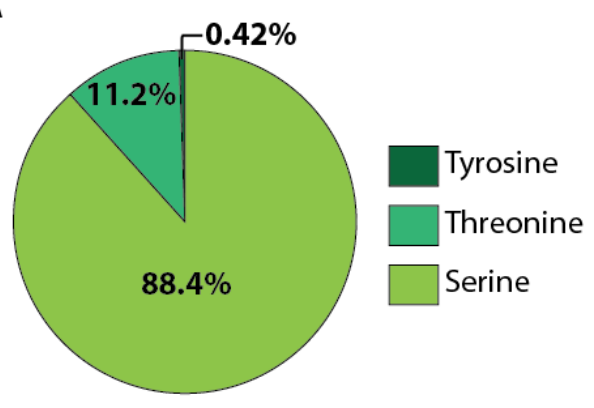

C

논

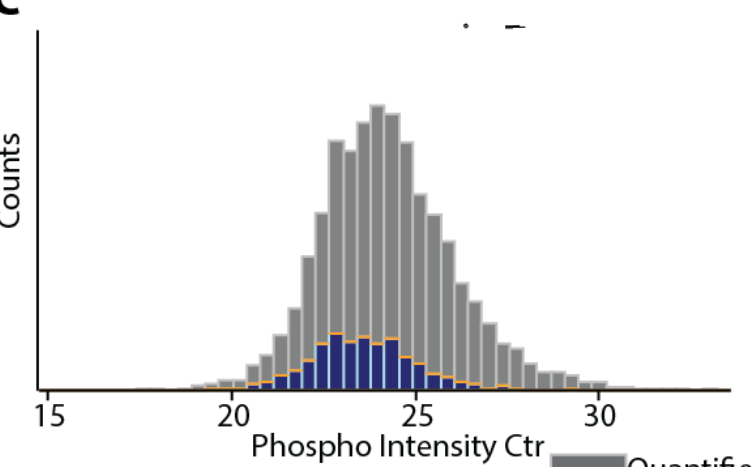

Quantified proteins

Phosphorylated proteins

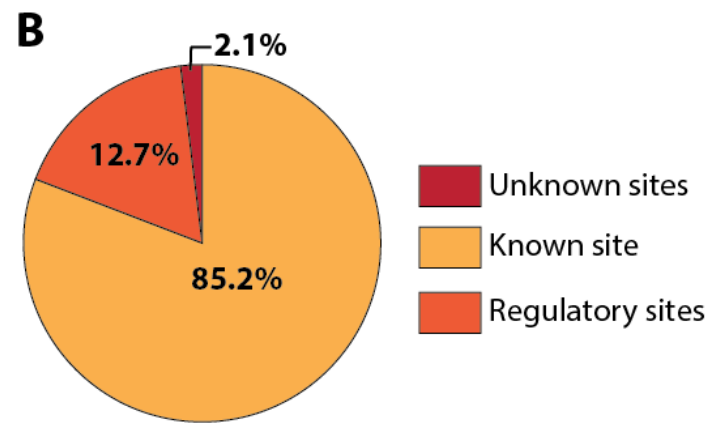

D

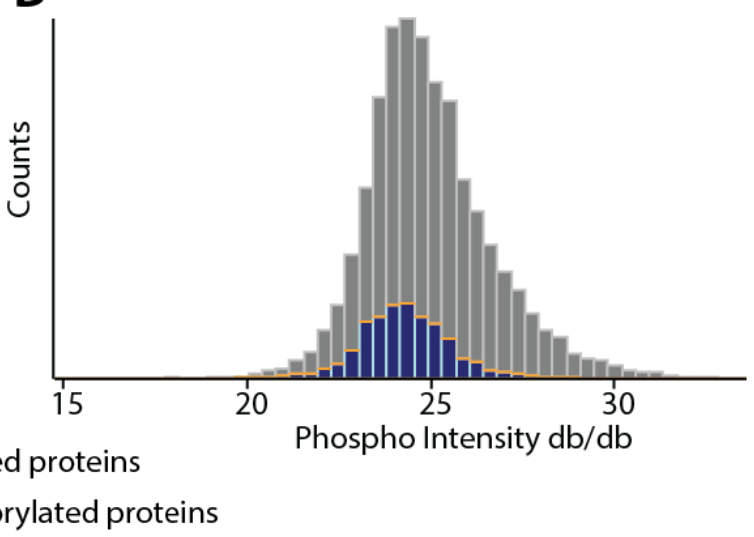

$\mathbf{E}$

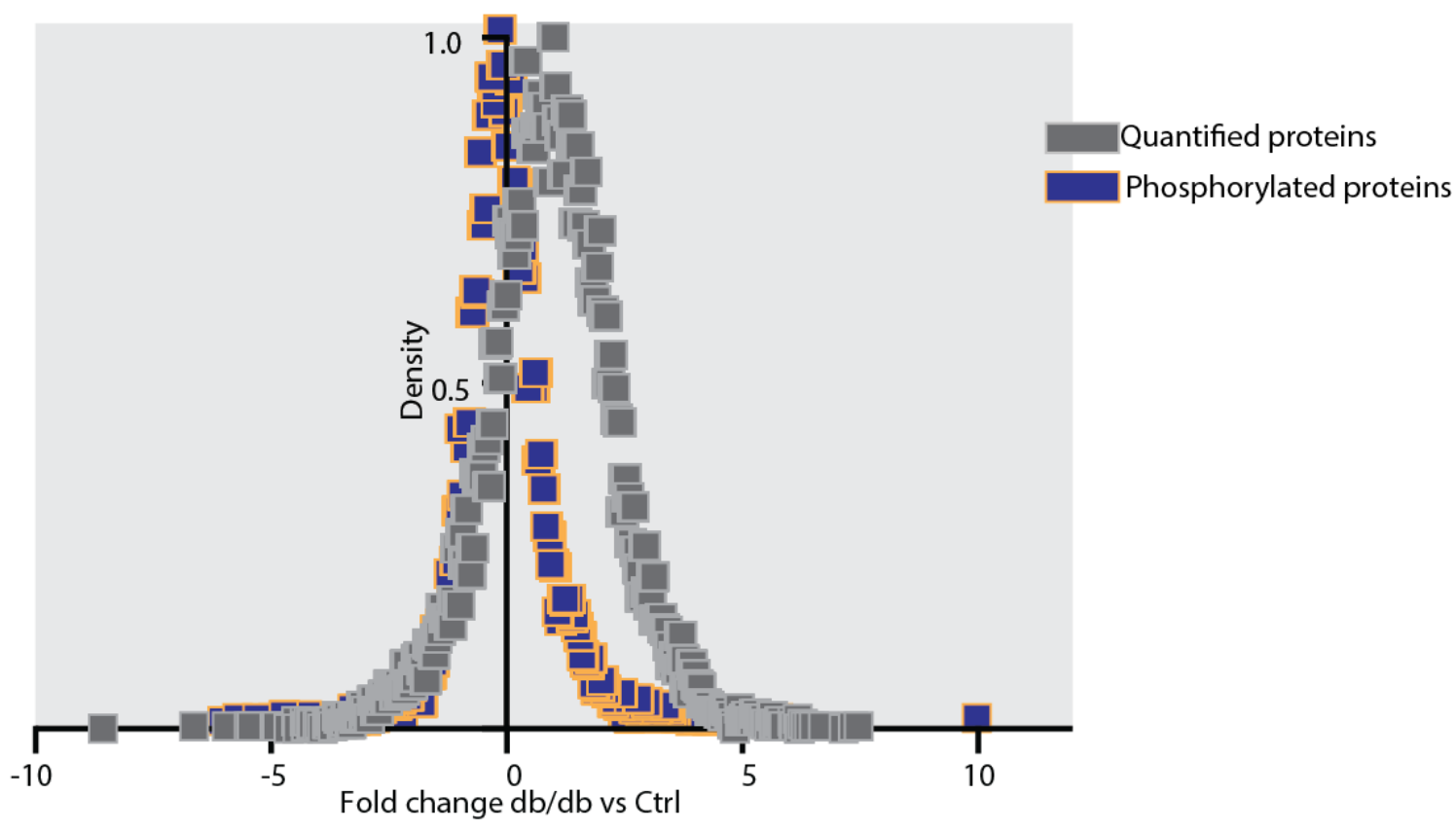


Figure S4. Comparison of proteins significantly modulated in $\mathrm{db} / \mathrm{db}$ islets, with different T2D literature-derived datasets. A) Venn diagram showing the overlap between the differentially expressed proteins derived by our and other proteomic approaches, as indicated. B) Multi-scatterplot showing the high correlation between fold changes of T2D-modulated proteins identified by different proteomic approaches. C) ER misfolded protein processing and degradation processes are schematically represented. Orange and red proteins are upregulated only in our $\mathrm{db} / \mathrm{db}$ dataset and in at least 3 different T2D datasets respectively. D) Heatmap of protein expression level of genes significantly modulated in $\mathrm{db} / \mathrm{db}$ islets and found to be significantly associated with T2D by GWAS studies.

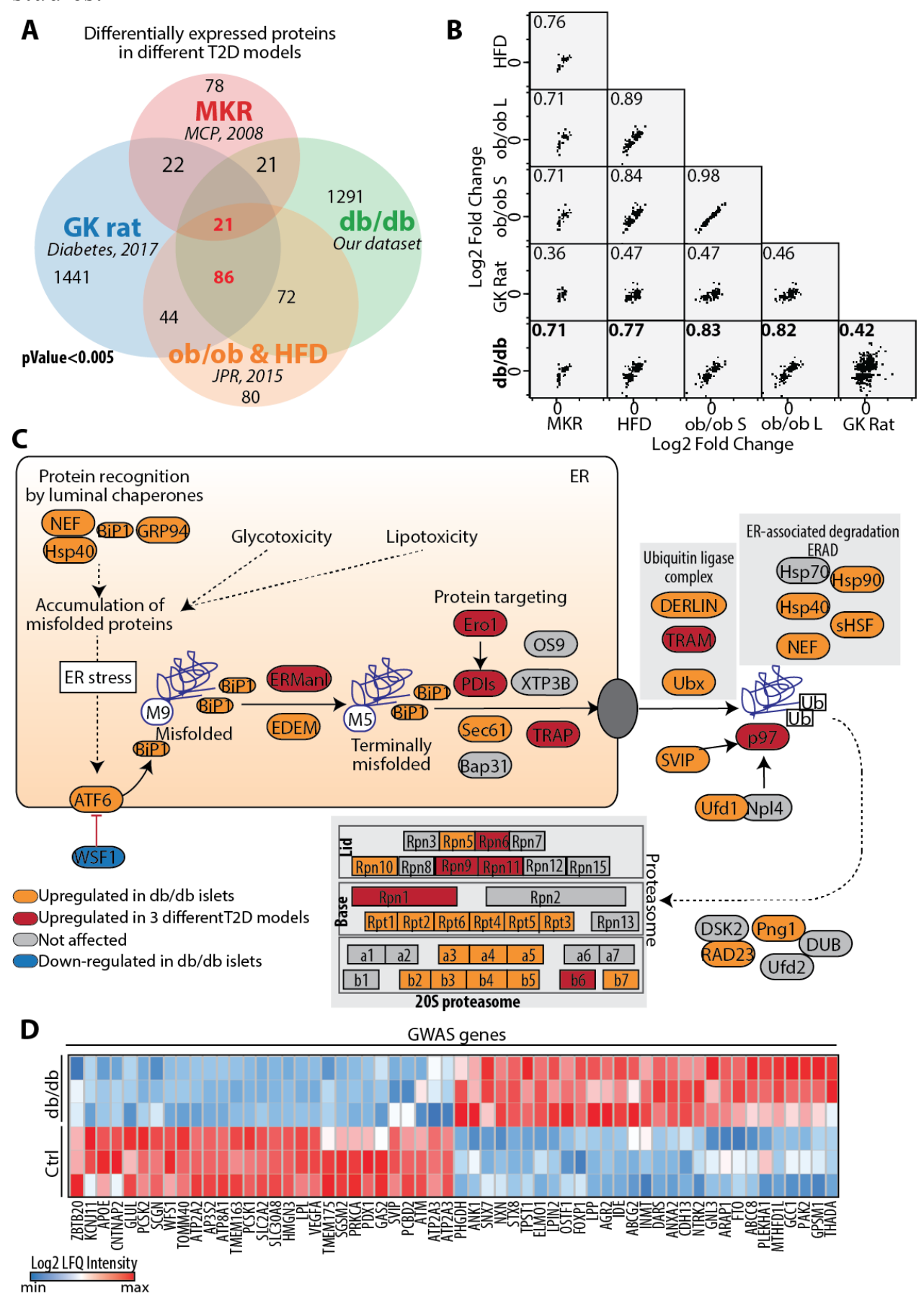


Figure S5. Proteomic comparison of islets derived from different T2D models. A-B-C-D) Plots showing the correlation between our dataset and previously published proteomic datasets of islets from the indicated T2D murine and rat models (El Ouaamari et al., 2015; Hou et al., 2017; Lu et al., 2008). E) Heatmap of the 107 proteins significantly modulated in islets from at least three different T2D models.
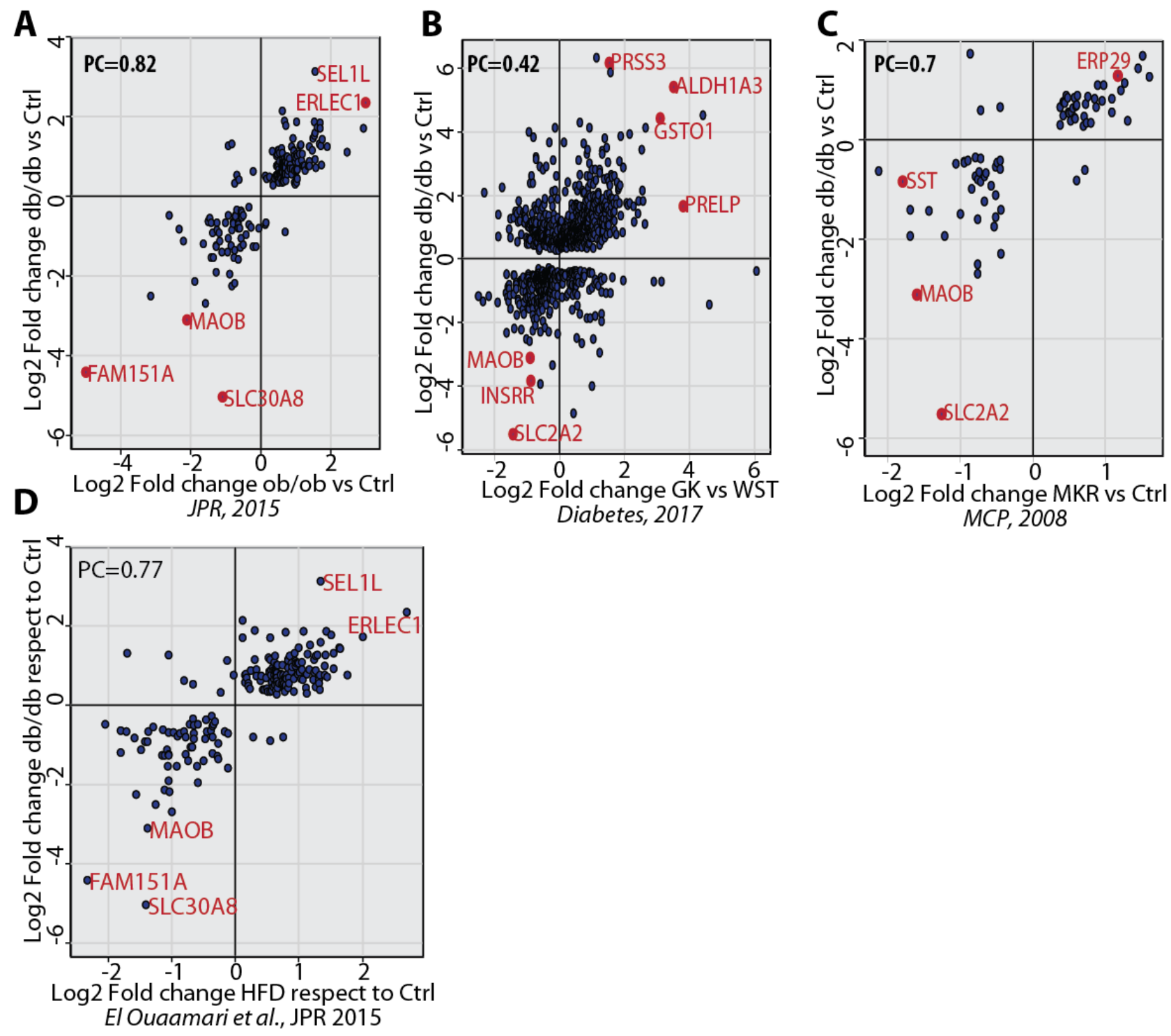

$\mathbf{E}$ 107 "core"T2D proteins

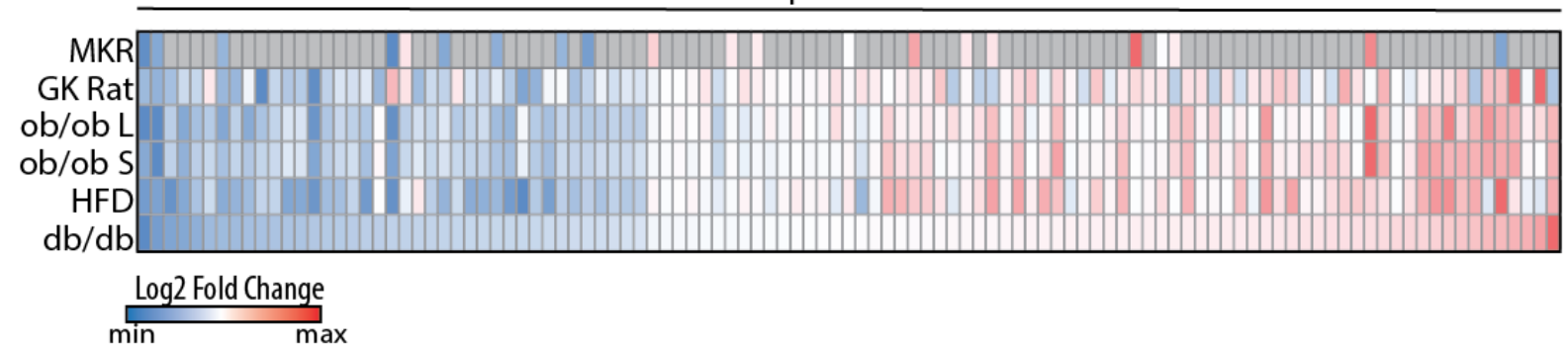


bioRxiv preprint doi: https://doi.org/10 1101/439638; this version posted October 11,2018 . The copyright holder for this preprint (which was not certified by peer review) is the author/funder, who has granted bioRxiv a license to display the preprint in perpetuity. It is made available under aCC-BY-NC-ND 4.0 International license.

Figure S6. Identification of significantly modulated proteins and phosphosites. Statistically significantly up- and down-regulated proteins (A) and phosphosites (B) were identified by t-test (Benjamin Hochberg FDR $<0.07$; S0 0.1 ) and represented as scatterplot. Each dot represents one protein (A) or one phosphosites (B). C) Enrichment and significance of GO-annotations, KEGG pathways and Keywords among the significantly modulated proteins.

A

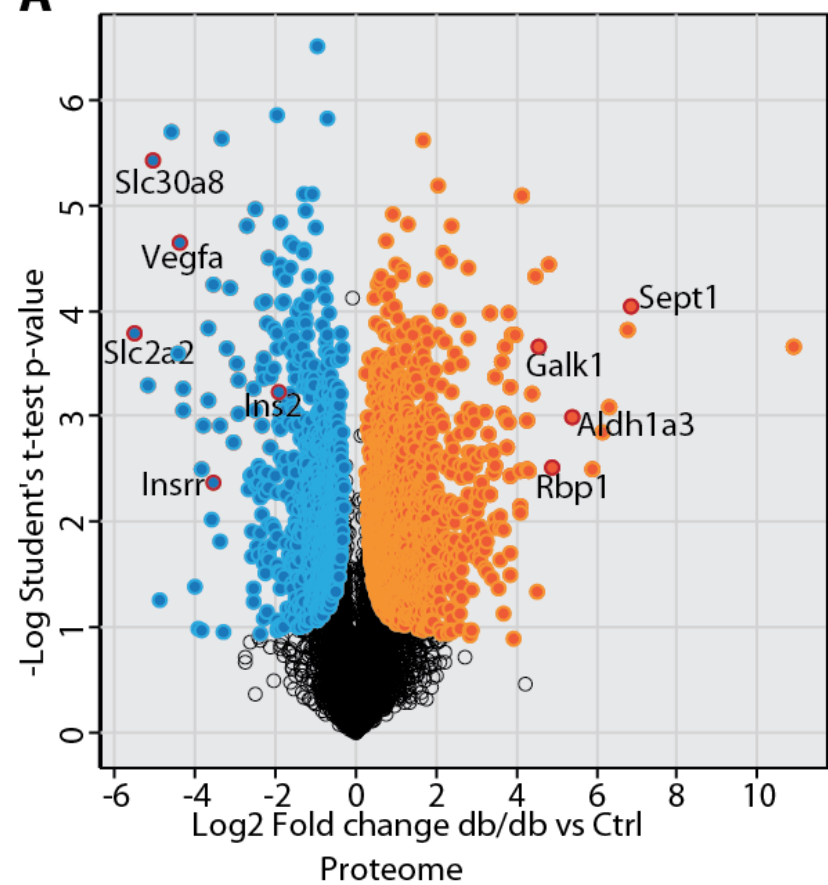

B

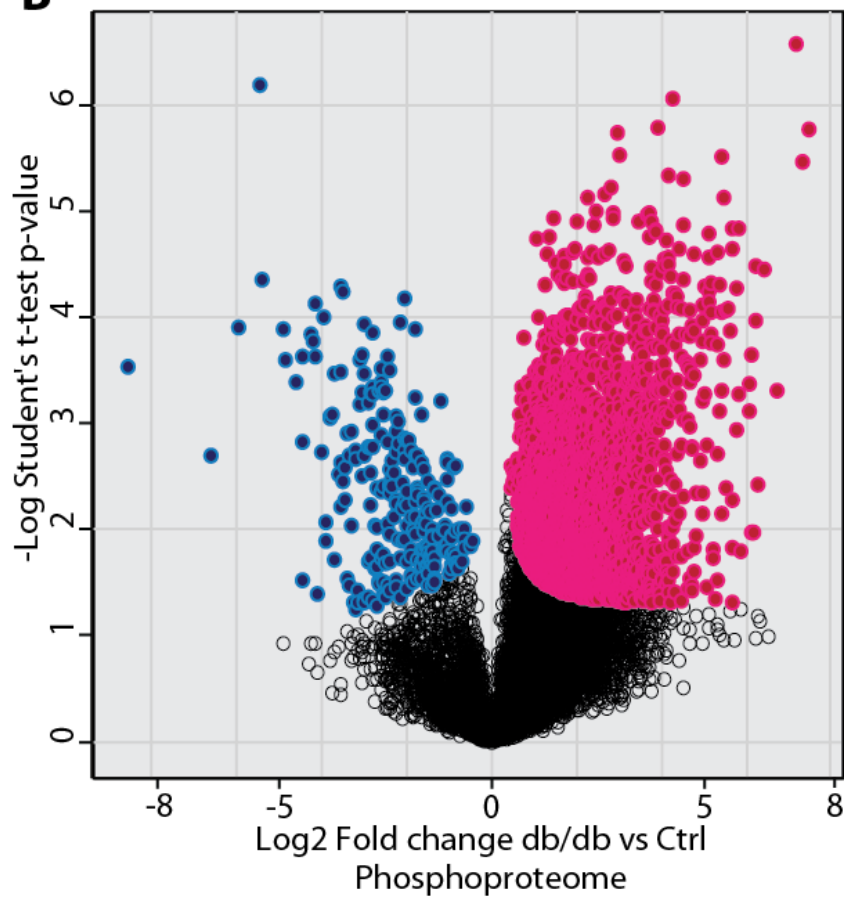

C

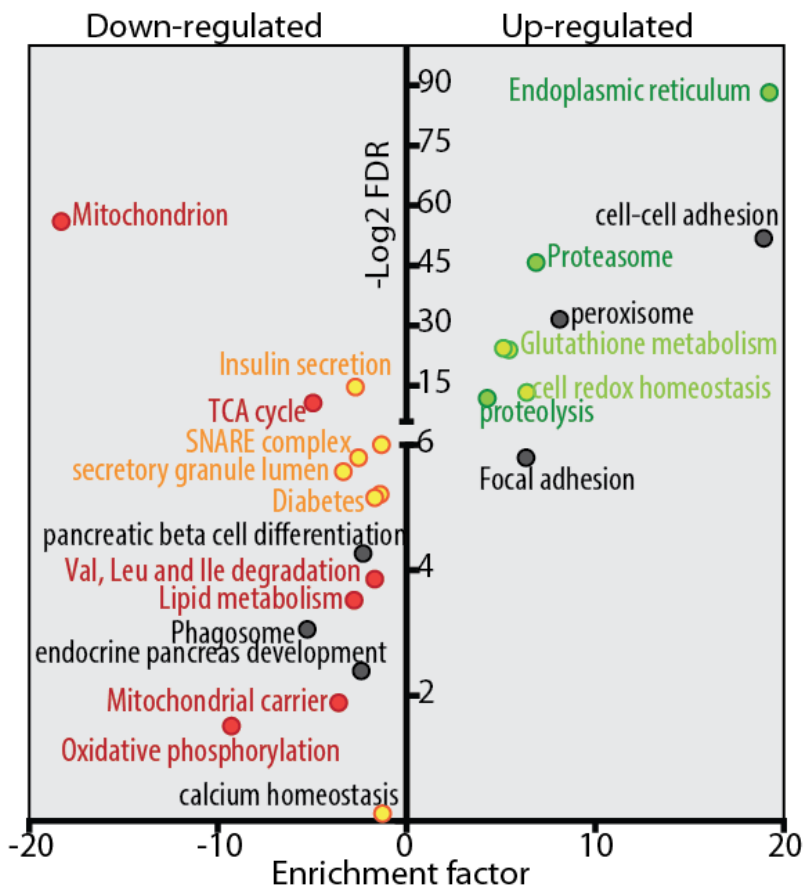


Figure S7. Key biological processes are regulated at the proteome and phosphoproteome levels. A) Pie chart indicating the percentage of positively and negatively modulated proteins (A), phosphosites (B) and phosphoproteins with at least one significantly modulated phosphopeptide (C). D) Percentage of phosphorylation sites with protein expression level quantified or not (left) and comparison between protein expression and phosphorylation levels for the $82 \%$ phosphorylation sites (right). Proteins and phosphorylation sites were considered regulated according to t-test analysis $(\mathrm{FDR}<0.07$; $\mathrm{S} 0=0.1)$. E) Plot showing the comparison of protein expression and phosphorylation level changes for the 39\% phosphosites in (D, right). F) Clusters of GO term and Kegg pathways enriched in the subset of genes significantly up and down-regulated at the proteome and phosphoproteome level are represented as nodes of a co-citation network. Node size is correlated to the frequency of co-citation of each term with "islets" in literature abstract. Edge thikness is proportial to the "co-citation score".

A

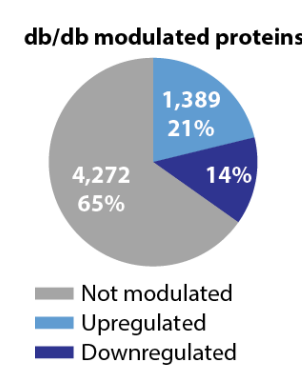

D

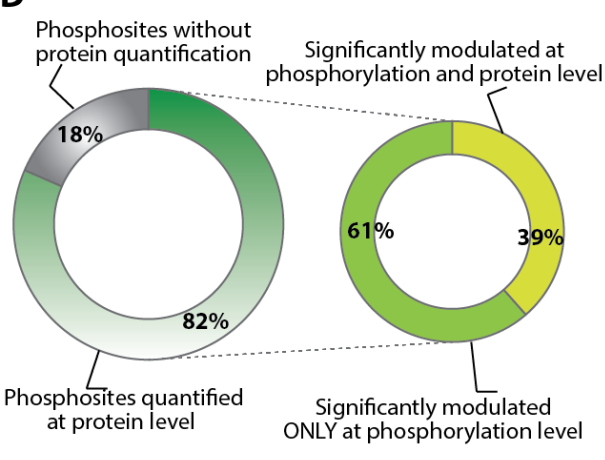

$\mathbf{F}$
B db/db modulated phosphosites $C$

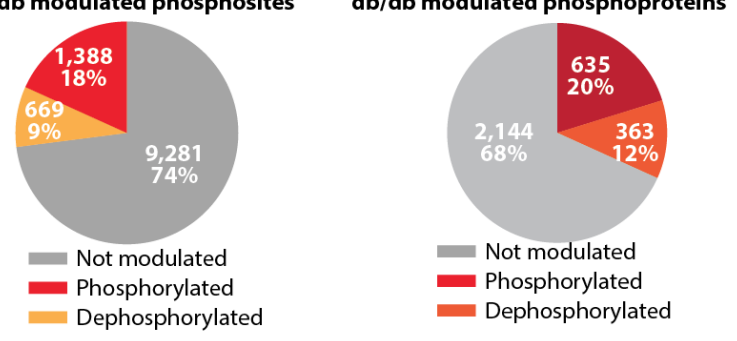

\section{E}

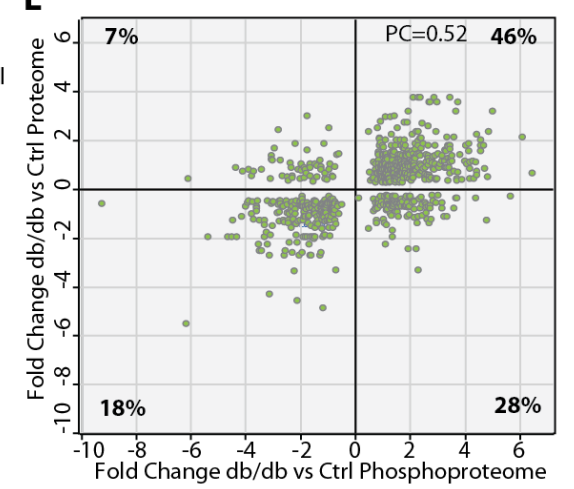

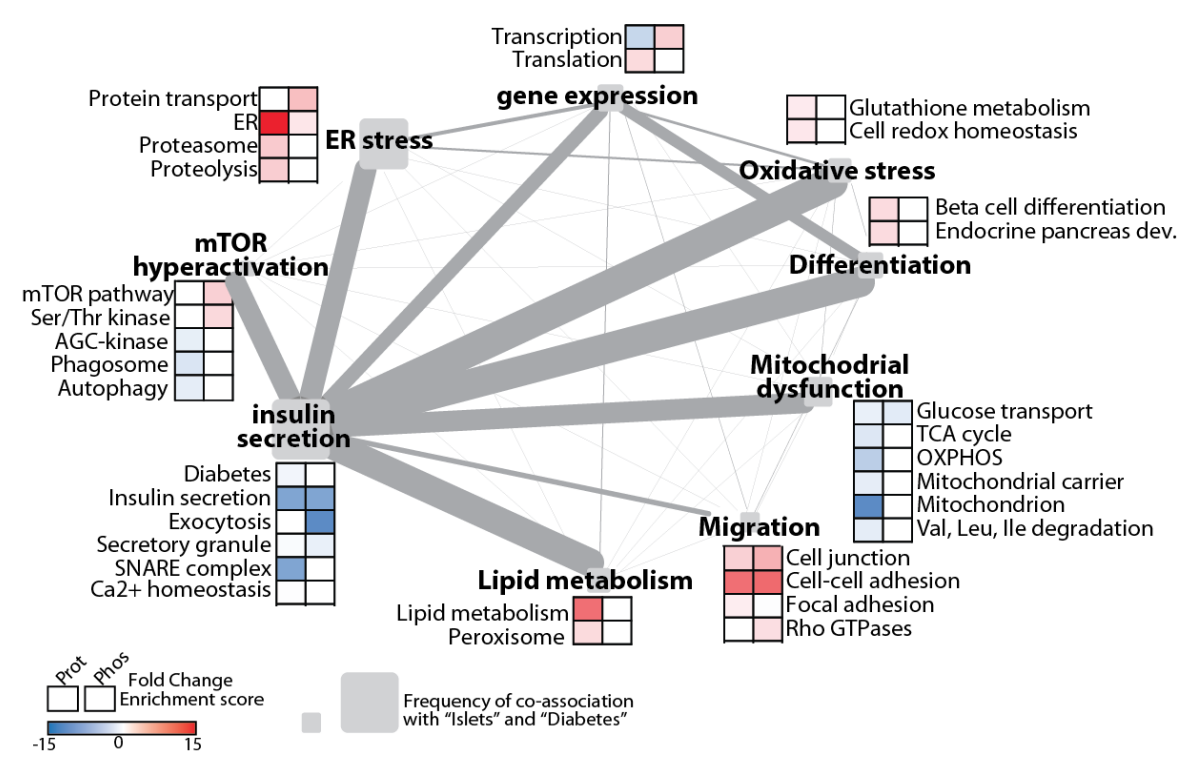


Figure S8. PDX1 phosphorylation in $\mathbf{d b} / \mathbf{d b}$ islets. Intensity of the PDX1 phosphopeptide containing S269 (phosphoproteome dataset) normalized to the total protein intensity of the PDX1 protein (LFQ from proteome).

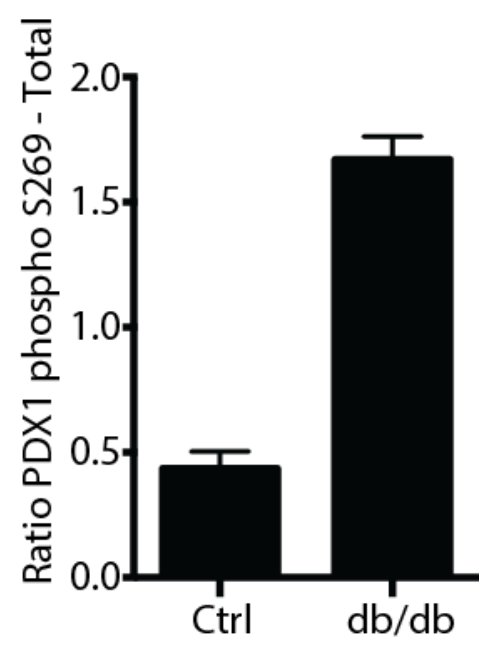


Figure S9. Reproducibility of biological replicates of proteome and phosphoproteome of glucose-treated INS1e cells. Heat map showing the Pearson correlation coefficients between the different biological replicates in the proteome $(\mathbf{B})$ and phosphoproteome $(\mathbf{C})$ datasets.

A
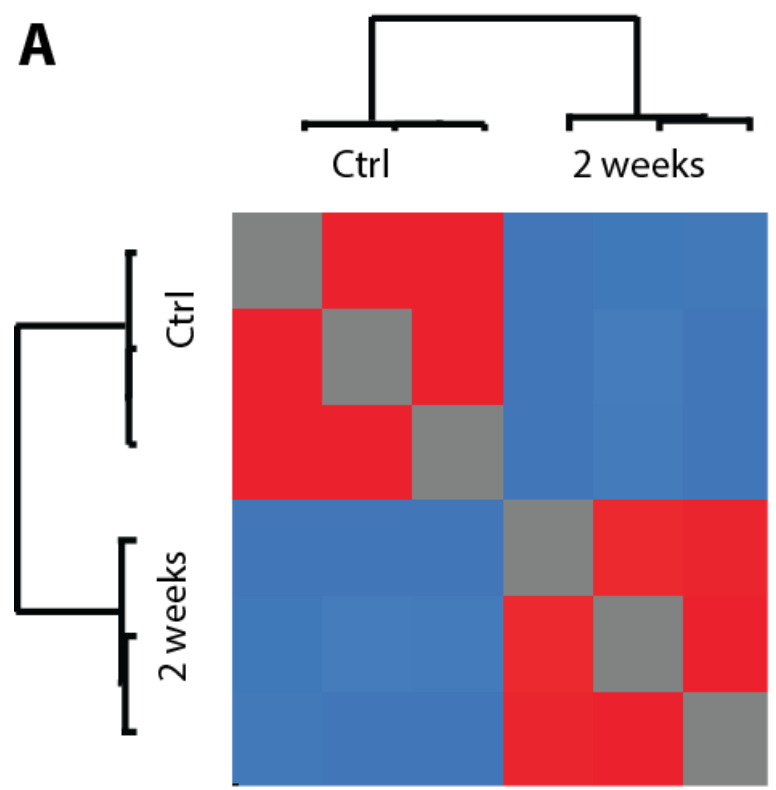

B

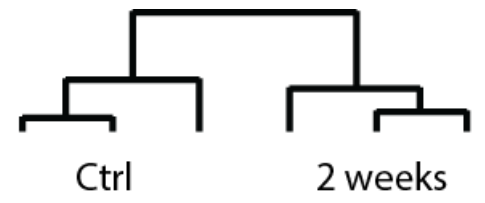

Pearson Correlation 0.90 .95 
bioRxiv preprint doi: https://doi.org/10.1101/439638; this version posted October 11,2018 . The copyright holder for this preprint (which was not certified by peer review) is the author/funder, who has granted bioRxiv a license to display the preprint in perpetuity. It is made available under aCC-BY-NC-ND 4.0 International license.

Figure S10. Proteomic comparison of islets derived from different T2D models. A) Indirect immunofluorescent analysis of GSK3 S9 (P), GLUT2, PDX1, Phalloidin and DAPI. B) Amount of secreted insulin (a.u.) after drug and glucose stimulation measured with an Elisa assay. Median and s.d. of triplicates are shown as a bar graph.

A

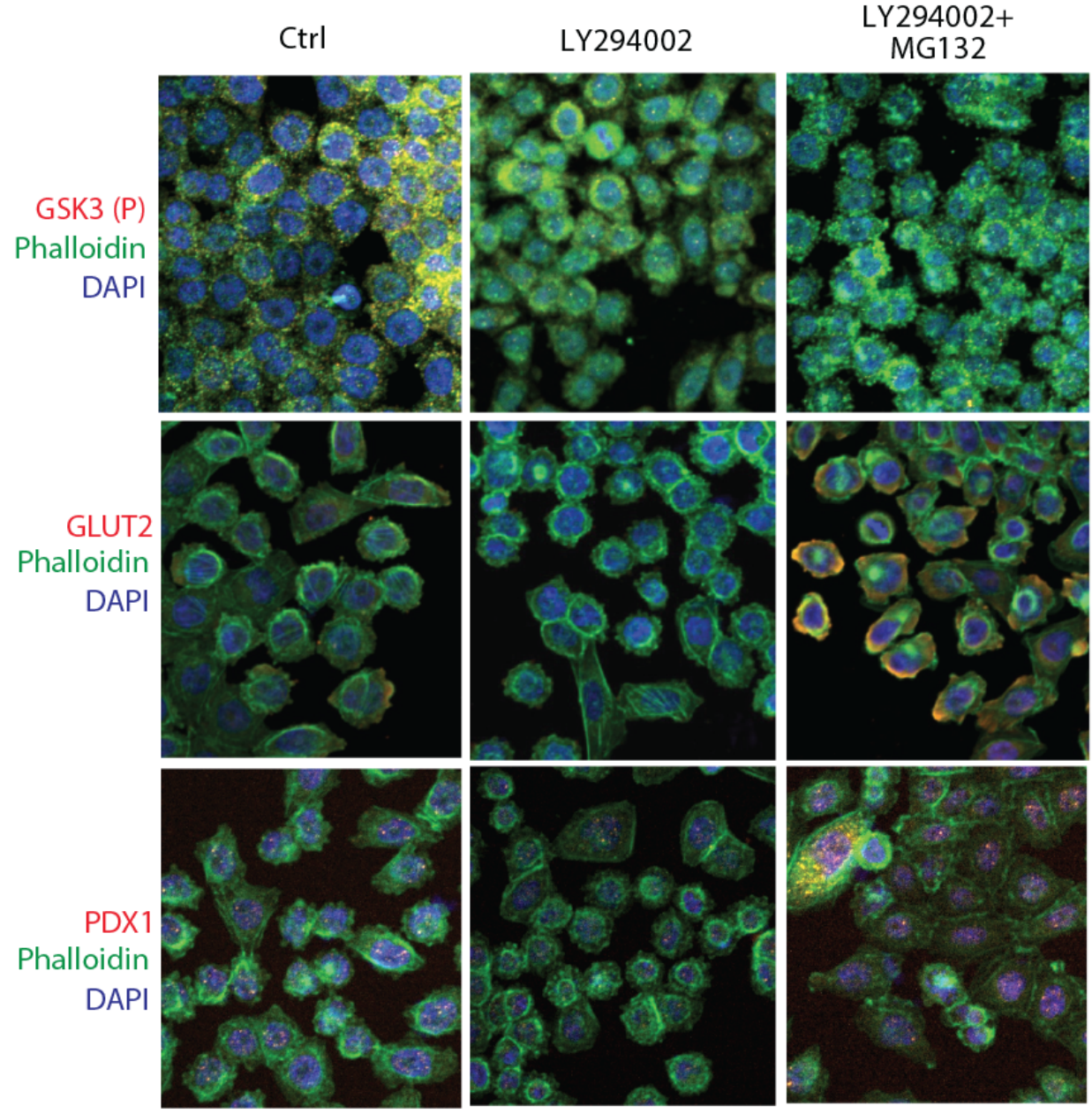

B

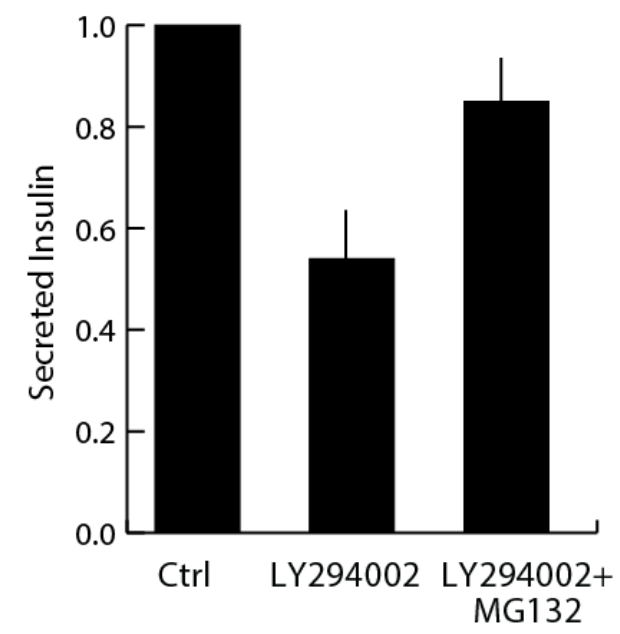




\section{Supplementary methods}

\section{Proteome and phosphoproteome data processing}

Raw mass spectrometry data were analyzed in the MaxQuant environment (Cox and Mann, 2008), version 1.5.1.6, employing the Andromeda engine for database search. MS/MS spectra were matched against the Mus Murine UniProt FASTA database (September 2014), with an FDR of $<1 \%$ at the level of proteins, peptides and modifications. Enzyme specificity was set to trypsin, allowing for cleavage N-terminal to proline and between aspartic acid and proline. The search included cysteine carbamidomethylation as a fixed modification, and N-terminal protein acetylation, oxidation of methionine and phosphorylation of serine, threonine tyrosine residue (STY) as variable modifications. Label free proteome analysis was performed in MaxQuant. For proteome and phosphoproteome analysis, where possible, the identity of peptides present but not sequenced in a given run was obtained by transferring identifications across liquid chromatography (LC)-MS runs ('match between runs'). For phosphopeptide identification, an Andromeda minimum score and minimum delta score threshold of 40 and 17 were used, respectively. Up to three missed cleavages were allowed for protease digestion and peptides had to be fully tryptic.

\section{Proteome and phosphoproteome bioinformatics data analysis}

Bioinformatic analysis was performed in the Perseus software environment (Tyanova et al., 2016). Statistical analysis of proteome and phosphoproteome was performed on logarithmized intensities for those values that were found to be quantified in any experimental condition. Phosphoproteome intensities were normalized by subtracting the median intensity of each sample. To identify significantly modulated proteins and phosphopetides across two different conditions, we performed a Student t-Test with a permutation-based FDR cutoff of 0.07 and $\mathrm{S} 0=0.1$. Categorical annotation was added in Perseus in the form of GO biological process (GOBP), molecular function (GOMF), and cellular component (GOCC), KEGG pathways and kinase substrate motifs (extracted from HPRD). Concerning the kinase substrate motifs, we performed a 1D annotation enrichment analyses to identify statistically significant enriched kinase-substrates motifs in $\mathrm{db} / \mathrm{db}$ islets (Cox and Mann, 2012). Multiple hypothesis testing was controlled by using a Benjamini-Hochberg FDR threshold of 0.05. Then for each kinase-substrate motif the corresponding $\mathrm{pValue}$ and score are assigned. While a score near 1 indicates a positive enrichment, a score near -1 means a negative enrichment of the category.

\section{Combining proteome and phosphoproteome data with a prior knowledge signaling network}

This strategy has been previously developed and applied by our group (Sacco et al., 2012; Sacco et al., 2016). Kinase-substrate relationships were extracted by PhosphositePlus (Kinase-substrate dataset) and SIGNOR (Hornbeck et al., 2015; Perfetto et al., 2016) and were mapped onto the complete human proteome. Then the network was first filtered to maintain only relationships between proteins that were identified in our proteomic analysis of $\mathrm{db} / \mathrm{db}$ islets or INS1e cells. This network was used as a scaffold to overlay the changes at the phosphoproteome level. Next the network was filtered according to the following rules: i) "leaf nodes" (connected only by one edge) whose phosphorylation was not quantified in our phospho- proteomics approach were excluded; ii) "Leaf nodes" whose phosphorylation was not modulated in $\mathrm{db} / \mathrm{db}$ islets or hyperglycaemic INS1e cells were also excluded and iii) those residues (edges) whose phosphorylation status was not supported by our 
experimental data were eliminated. This filtering procedure yielded a much simpler network that was easier to analyse. For those proteins having multiple regulatory residues and whose phosphorylation was strongly changed in opposite direction, we considered only those sites quantified in a singly phosphorylated peptide (multiplicity $=1$ ).

\section{Immunofluorescence microscopy}

Human pancreatic islets were treated with LY294002 with or without MG132. Cells were fixed for $10 \mathrm{~min}$ in 4\% paraformaldehyde (EM Sciences), washed with PBS, 0.5\% Triton X-100, and permeabilized with blocking solution $(0.1 \%$ Triton, $10 \%$ fetal calf serum) for $30 \mathrm{~min}$. Cells were incubated, in blocking solution, with anti-GSK3 (P) (RRID:AB_10013750), anti-GLUT2, anti-PDX1 and anti FITC phalloidin (RRID:AB_2315147) for $1 \mathrm{~h}$ at room temperature. Cells were rinsed in PBS and incubated with secondary antibody for 30 minutes. Cells were stained with 4',6-diamidino-2phenylindole in PBS, $0.1 \%$ Triton for $5 \mathrm{~min}$ at room temperature. Cells were analyzed by indirect immunofluorescence microscopy. 


\section{Supplementary References}

Cox, J., and Mann, M. (2008). MaxQuant enables high peptide identification rates, individualized p.p.b.-range mass accuracies and proteome-wide protein quantification. Nat Biotechnol 26, 13671372.

Cox, J., and Mann, M. (2012). 1D and 2D annotation enrichment: a statistical method integrating quantitative proteomics with complementary high-throughput data. BMC Bioinformatics 13 Suppl $16, \mathrm{~S} 12$.

El Ouaamari, A., Zhou, J.Y., Liew, C.W., Shirakawa, J., Dirice, E., Gedeon, N., Kahraman, S., De Jesus, D.F., Bhatt, S., Kim, J.S., et al. (2015). Compensatory Islet Response to Insulin Resistance Revealed by Quantitative Proteomics. J Proteome Res 14, 3111-3122.

Hornbeck, P.V., Zhang, B., Murray, B., Kornhauser, J.M., Latham, V., and Skrzypek, E. (2015). PhosphoSitePlus, 2014: mutations, PTMs and recalibrations. Nucleic Acids Res 43, D512-520.

Hou, J., Li, Z., Zhong, W., Hao, Q., Lei, L., Wang, L., Zhao, D., Xu, P., Zhou, Y., Wang, Y., et al. (2017). Temporal Transcriptomic and Proteomic Landscapes of Deteriorating Pancreatic Islets in Type 2 Diabetic Rats. Diabetes 66, 2188-2200.

Lu, H., Yang, Y., Allister, E.M., Wijesekara, N., and Wheeler, M.B. (2008). The identification of potential factors associated with the development of type 2 diabetes: a quantitative proteomics approach. Mol Cell Proteomics 7, 1434-1451.

Perfetto, L., Briganti, L., Calderone, A., Cerquone Perpetuini, A., Iannuccelli, M., Langone, F., Licata, L., Marinkovic, M., Mattioni, A., Pavlidou, T., et al. (2016). SIGNOR: a database of causal relationships between biological entities. Nucleic Acids Res 44, D548-554.

Sacco, F., Gherardini, P.F., Paoluzi, S., Saez-Rodriguez, J., Helmer-Citterich, M., Ragnini-Wilson, A., Castagnoli, L., and Cesareni, G. (2012). Mapping the human phosphatome on growth pathways. Mol Syst Biol 8, 603.

Sacco, F., Humphrey, S.J., Cox, J., Mischnik, M., Schulte, A., Klabunde, T., Schafer, M., and Mann, M. (2016). Glucose-regulated and drug-perturbed phosphoproteome reveals molecular mechanisms controlling insulin secretion. Nat Commun 7, 13250.

Tyanova, S., Temu, T., Sinitcyn, P., Carlson, A., Hein, M.Y., Geiger, T., Mann, M., and Cox, J. (2016). The Perseus computational platform for comprehensive analysis of (prote)omics data. Nat Methods 13, 731-740. 